\title{
On Recent Progress in Modelling and Simulations of Multi-scale Transfer of Mass, Momentum and Particles in Bio-medical Applications
}

\author{
Saša Kenjereš ${ }^{1,2}$
}

Received: 13 January 2015 / Accepted: 8 October 2015 / Published online: 3 November 2015

(C) The Author(s) 2015. This article is published with open access at Springerlink.com

\begin{abstract}
We present a short overview of some of our most recent work that combines the mathematical modeling, advanced computer simulations and state-of-the-art experimental techniques of physical transport phenomena in various bio-medical applications. In the first example, we tackle predictions of complex blood flow patterns in the patient-specific vascular system (carotid artery bifurcation) and transfer of the so-called "bad" cholesterol (low-density lipoprotein, LDL) within the multi-layered artery wall. This two-way coupling between the blood flow and corresponding mass transfer of LDL within the artery wall is essential for predictions of regions where atherosclerosis can develop. It is demonstrated that a recently developed mathematical model, which takes into account the complex multilayer arterial-wall structure, produced LDL profiles within the artery wall in good agreement with in-vivo experiments in rabbits, and it can be used for predictions of locations where the initial stage of development of atherosclerosis may take place. The second example includes a combination of pulsating blood flow and medical drug delivery and deposition controlled by external magnetic field gradients in the patient specific carotid artery bifurcation. The results of numerical simulations are compared with own PIV (Particle Image Velocimetry) and MRI (Magnetic Resonance Imaging) in the PDMS (silicon-based organic polymer) phantom. A very good agreement between simulations and experiments is obtained for different stages of the pulsating cycle. Application of the magnetic drug targeting resulted in an
\end{abstract}

Saša Kenjereš

S.Kenjeres@tudelft.nl

1 Transport Phenomena Section, Department of Chemical Engineering, Faculty of Applied Sciences,

Delft University of Technology, Julianalaan 136, 2628 BL Delft, The Netherlands

2 J. M. Burgers Centre for Fluid Mechanics, Julianalaan 136, 2628 BL Delft, The Netherlands 
increase of up to ten fold in the efficiency of local deposition of the medical drug at desired locations. Finally, the LES (Large Eddy Simulation) of the aerosol distribution within the human respiratory system that includes up to eight bronchial generations is performed. A very good agreement between simulations and MRV (Magnetic Resonance Velocimetry) measurements is obtained. Magnetic steering of aerosols towards the left or right part of lungs proved to be possible, which can open new strategies for medical treatment of respiratory diseases.

Keywords Blood flow $\cdot$ LDL transfer - Aerosol distribution · Magnetization force

\section{Introduction}

Physical transport phenomena (integrated studies of mass, momentum and energy) within biological systems play a crucial role in the functioning of cells, tissues and organs. Some changes in biological transport processes can trigger a complex chain of bio-chemical reactions leading to any of a number of diseases. Typical examples include atherosclerosis, asthma, allergies, cancer, etc. The proper understanding and advanced mathematical modeling of the basic mechanisms behind transport phenomena in living systems can make it possible to predict locations where the initial stage of various diseases will occur. Then, as a next step, various optimizations of the medical drug delivery aimed precisely towards these regions can be performed. This can lead to a significant increase of the local medical drug concentration in the proximity of diseased areas and consequently, to reduction of side effects. In addition to these 'direct' medical drug delivery applications, numerous applications can be found also in bio-medical engineering dealing with development, design and optimization of various medical assisting devices, such as artificial heart (ventricular assist device), artificial lungs, artificial kidneys (dialysis) machines etc.

There are many challenges in dealing with transport phenomena in living systems. The typical length scales $(\mathscr{L})$ of the major transport phenomena in humans cover an immense range. Starting from the whole human body with $\mathscr{L} \approx \mathscr{O}\left(10^{0}\right) \mathrm{m}$, organs $\mathscr{L} \approx \mathscr{O}\left(10^{-1}\right) \mathrm{m}$, cells $\mathscr{L} \approx \mathscr{O}\left(10^{-6}\right) \mathrm{m}$, and finally, proteins $\mathscr{L} \approx \mathscr{O}\left(10^{-8}\right) \mathrm{m}$. It is obvious that no single transport mechanism can function equally efficiently over these length scales. Diffusive transport can be very efficient for the small length scales, but is replaced by convection for transport of molecules over larger distances. For example diffusion is most important for small molecules such as oxygen (with estimated Peclet number of $\mathrm{Pe} \approx \mathscr{O}\left(10^{-2}\right)$ ), whereas convection is the dominant mechanism for transport of larger entities such as cells (with estimated $\mathrm{Pe} \approx \mathscr{O}\left(10^{3}\right)$ ), Truskey et al. [1]. The range of typical time scales $(\mathscr{T})$ is also immense: from $\mathscr{T} \approx \mathscr{O}(1)$ s, for typical cardiac cycle (heartbeat), up to $\mathscr{T} \approx \mathscr{O}\left(10^{8}\right)$ s (ten years) for development of atherosclerosis.

In addition to an immense range of length- and time-scales, the transport phenomena in bio-medical applications involve complex multi-physics interactions that include mass and heat transfer, bio-chemical reactions, phase changes, fluid dynamics, complex fluids (blood rheology), fluid-structure interactions, electromagnetism etc. To deal with such complex problems, a multi-disciplinary approach is required, which combines biology, medicine, physics, applied mathematics, bio-medical, mechanical and chemical engineering, imaging and computer sciences. In the present paper, we will address some of our most recent achievements in mathematical modeling and combined numerical and experimental studies 


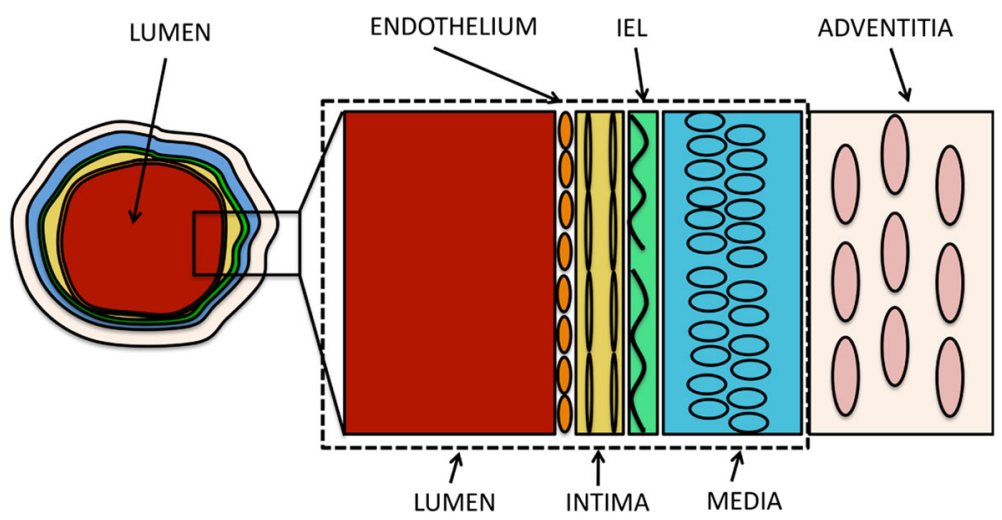

Fig. 1 Schematic representation of the multi-layered structure of an artery wall (not to scale): the artery interior (lumen), the arterial wall (endothelium, intima, internal elastic layer, media and adventitia). The typical thickness of these layers is: endothelium $(\approx 2 \mu \mathrm{m})$, intima $(\approx 10 \mu \mathrm{m})$, IEL $(\approx 2 \mu \mathrm{m})$, media $(\approx 200$ $\mu \mathrm{m})$ and adventitia $(\approx 200 \mu \mathrm{m})$

dealing with combined mass- and momentum-transfer of blood flow in anatomically realistic human arteries and of the air flow and aerosol distribution in the human respiratory system.

\section{Modeling and Simulation of the Initial Stage of Atherosclerosis}

Atherosclerosis (one of the most common cardiovascular diseases) is a process of the accumulation of fatty plaques within the artery wall. It primarily takes place at curvatures and bifurcations of large and medium size arteries, e.g. the coronary or carotid arteries. The rupture of the soft fatty plaque and its release into the blood stream leads to heart attack or stroke. Based on some recent reports of the World Health Organization ${ }^{1}$, it is estimated that 17.3 million people died from the cardiovascular diseases in 2008, and that by the year 2030, more than 23 million people will die of it annually. At present, it is accepted that atherosclerosis is a chronic inflammatory disease triggered by damage of the endothelium layer (the very first layer of the arterial wall) and by an increased accumulation of the "bad" cholesterol (i.e. low-density-lipoprotein, LDL) within the interior of the artery wall. It is obvious that an early prediction of the possible locations where atherosclerosis can develop can have huge impact in treatment of this disease, Tarbell [2]. Here, we will give a short overview of a recently developed mathematical model that combines solutions of the blood flow and LDL accumulation within the multi-layered arterial wall (shown in Fig. 1) of an anatomically realistic carotid artery bifurcation (shown in Fig. 2). It should be noted that there are differences in the characteristic length-scales from a few $\mathrm{cm}$ (length of the

${ }^{1}$ http://www.who.int 


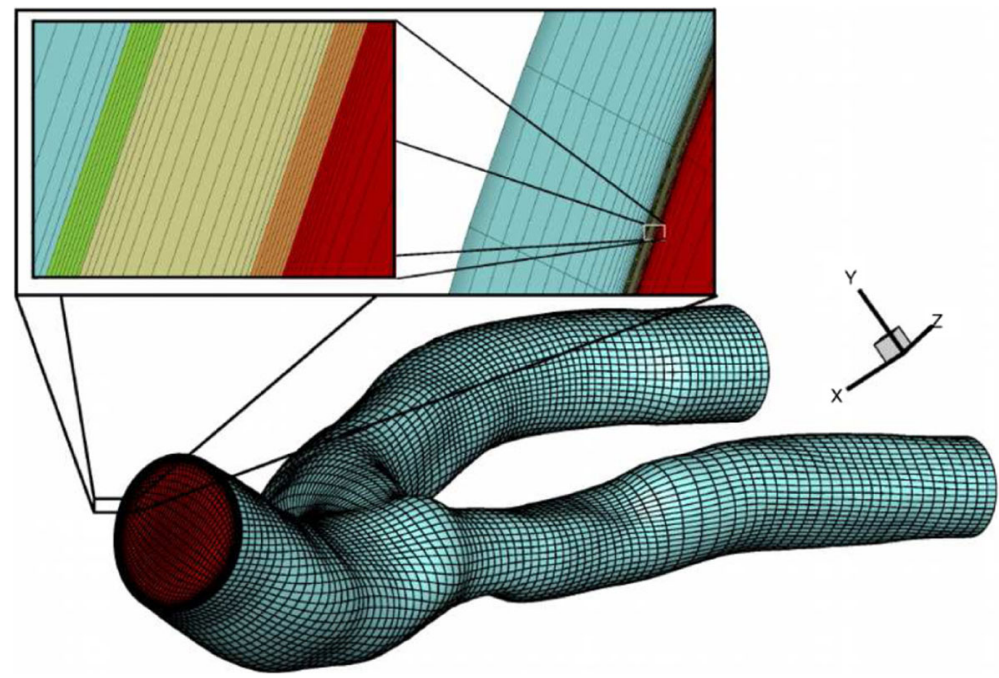

Fig. 2 Typical numerical mesh for the patient-specific carotid artery, covering the artery interior and the multi-layered artery wall (indicated in Fig. 1)

artery) to a few $\mu \mathrm{m}$ (the typical thickness of endothelium layer). Equations for blood flow and LDL transfer within the lumen (inner part of artery that is occupied with flowing blood) are:

$$
\begin{aligned}
\nabla \cdot \mathbf{v} & =0 \\
\rho \frac{\partial \mathbf{v}}{\partial t}+\rho(\mathbf{v} \cdot \nabla) \mathbf{v} & =-\nabla p+\nabla(\mu \nabla \mathbf{v})
\end{aligned}
$$

where the LDL concentration is modeled with the standard convective-diffusive mass transport as

$$
\frac{\partial c}{\partial t}+\mathbf{v} \cdot \nabla c=\mathscr{D} \nabla^{2} c
$$

Here $\rho$ is the density, $\mu$ the dynamical viscosity and $\mathscr{D}$ the diffusion coefficient. Transport of the blood plasma and of the LDL concentration within the multi-layered arterial wall is modeled by combining the porous-layer and biological membranes approach and can be written as [3-5]:

$$
\begin{gathered}
\nabla \cdot\langle\mathbf{v}\rangle=0 \\
\frac{\rho}{\varepsilon} \frac{\partial\langle\mathbf{v}\rangle}{\partial t}+\frac{\rho}{\varepsilon}(\langle\mathbf{v}\rangle \cdot \nabla)\langle\mathbf{v}\rangle=-\frac{\nabla\langle p\rangle}{\varepsilon}+\nabla \cdot \frac{\mu}{\varepsilon} \nabla\langle v\rangle-\frac{\mu}{K}\langle\mathbf{v}\rangle+\sigma_{d} R T \nabla\langle c\rangle
\end{gathered}
$$

where " \langle\rangle " are volume-averaged variables, the $\varepsilon$ is the porosity (defined as a fraction of the void volume over the total volume; $\varepsilon=0$ for the totally blocked flow), $K$ is the permeability, $\sigma_{d}$ is the Staverman osmotic reflection term and $R$ is the universal gas constant and $T$ is the absolute temperature (in K). The third and fourth terms on the right side of the extended momentum Eq. 5 are the flow resistance imposed by porous medium (Darcy's law) and 
the osmotic pressure, respectively. The osmotic pressure term makes a two-way coupling between the blood plasma momentum and the LDL concentration. The volume-averaged equation for the LDL concentration can be written as:

$$
\frac{\partial\langle c\rangle}{\partial t}+\left(1-\sigma_{f}\right)\langle\mathbf{v}\rangle \cdot \nabla\langle c\rangle=\mathscr{D}_{e} \nabla^{2}\langle c\rangle+k_{r}\langle c\rangle
$$

where $\mathscr{D}_{e}$ is the effective diffusivity, the $\sigma_{f}$ is the Staverman filtration coefficient, and $k_{r}$ is the effective volumetric reaction rate coefficient. To have continuous mass transport across the different interfaces, the following boundary condition is used

$$
\left.\left[\left(1-\sigma_{f}\right) v_{n} c-\mathscr{D}_{e} \frac{\partial c}{\partial n}\right]\right|_{+}=\left.\left[\left(1-\sigma_{f}\right) v_{n} c-\mathscr{D}_{e} \frac{\partial c}{\partial n}\right]\right|_{-}
$$

where $v_{n}$ is the normal velocity to the interface. A detailed overview of the model parameters, which include density, diffusivity, viscosity, permeability, porosity and reflection coefficients for each individual layer, collected from various sources in the literature, are shown in Yang and Vafai [3] and Kenjereš and de Loor [6].

The multi-layered arterial wall layers are generated around the patient specific carotid artery geometry received from the Erasmus Medical Center in Rotterdam (the computed tomography (CT) scans), Fig. 2. The important geometrical features of the circumferentially averaged diameters of the common carotid artery (CCA) (the main branch before bifurcation), and of the internal (ICA) and of the external carotid artery (ICA) (branches after the bifurcation), are $6.5,4$ and $3.2 \mathrm{~mm}$, respectively. The total length of the segment of the carotid artery bifurcation is about $12 \mathrm{~cm}$. The numerical mesh is refined in the radial direction in the proximity of the interfaces between layers. In total, about $2 \times 10^{6}$ control volumes were sufficient to get grid-independent results, with a second-order linear upwind scheme used for the convective terms in the momentum and concentration equations, [7].

The boundary conditions are imposed to ensure the medically recorded mass-flow ratios through the ICA and ECA of $0.6: 0.4$, Groen et al. [8]. The characteristic transmural pressure gradient (a pressure difference between the interior and exterior of the artery) of 70 $\mathrm{mmHg}$ is specified to mimic a normal blood pressure, Fig. 3. At the inlet, a fully developed laminar velocity profile is imposed to give $\mathrm{Re}=105$.

The inlet value of the LDL concentration of $c_{0}=2.5 \mathrm{~mol} / \mathrm{m}^{3}$ (a normal value for a healthy person) is specified. We are interested in the long-time accumulation of LDL within the artery wall, so the system of equations given by Eqs. 1-6 is solved in a steady mode. The results are shown in Fig. 4, where contours of the wall-shear-stress (WSS) and of the nondimensional LDL concentration $\left(c / C_{0}\right)$ are plotted along the lumen/endothelium interface.

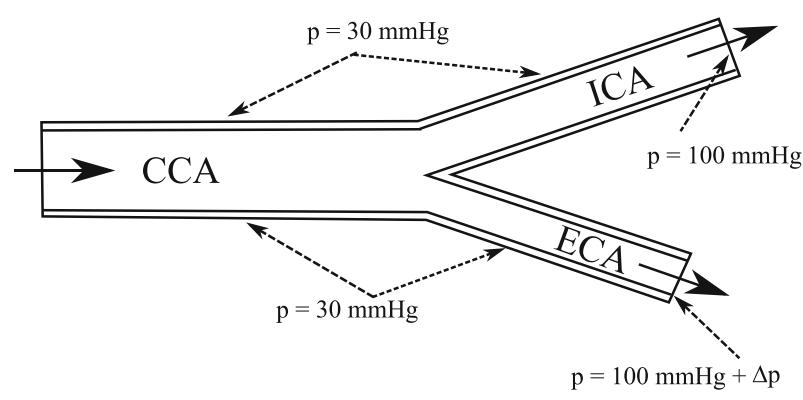

Fig. 3 Applied boundary conditions used for the carotid artery simulations. The steady flow conditions are considered with the inlet value of $\operatorname{Re}=105$, Kenjereš and de Loor [6] 


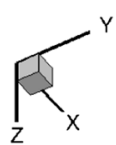

\section{CCA}
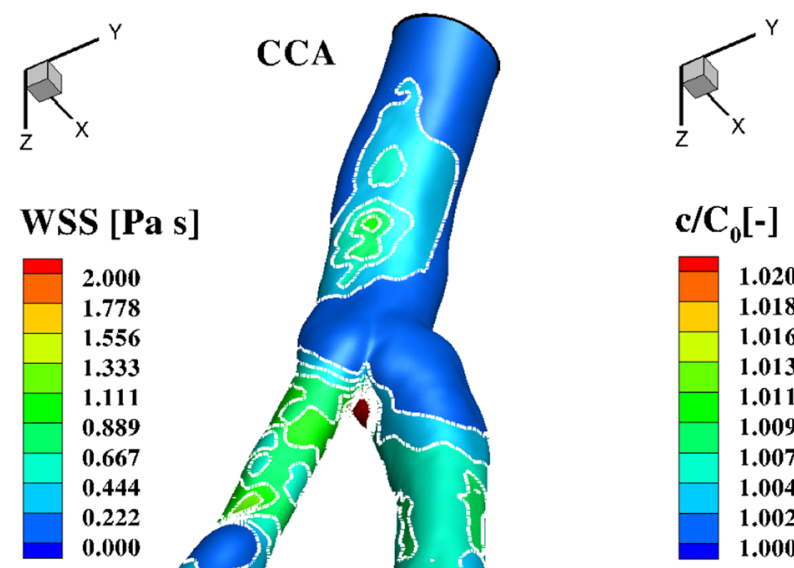

2.000

1.778

1.556

1.333

1.111

0.889

0.667

$\mathbf{0 . 4 4 4}$

0.222

0.000

(a)

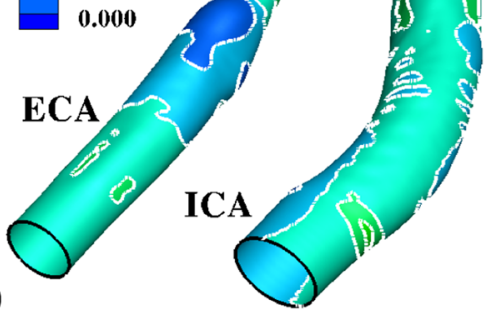

(b)

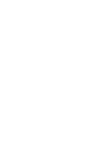

CCA

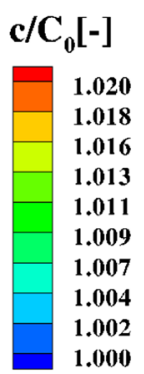

\section{ECA}

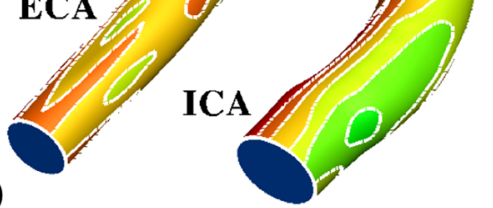

Fig. 4 Contours of the wall-shear-stress (WSS) (a) and of the non-dimensional LDL concentration (b) at the lumen/endothelium interface for the flow conditions defined in Fig. 3

We observe a correlation between the low WSS and high LDL concentration regions. The strong variation of the LDL concentration levels is observed in the proximity of bifurcation, confirming the importance of the underlying blood flow patterns on the mass transfer within the arterial wall, as shown in Kenjereš and de Loor [6]. Note that an increase of about $3 \%$ is obtained, confirming proper capturing of the concentration polarization. The standard convective-diffusive type of mass transfer is not able to predict such a mechanism. To capture polarization effects, it is crucial to implement biological membrane effects, as shown in Eq. 5. The locally increased values of the LDL concentration indicate locations where atherosclerosis may take place. Finally, the radial profiles of the LDL concentration at characteristic locations shown in Fig. 5 are compared with experimental results. These experiments were performed in the rabbit artery subjected to identical values of the transmural pressure of $70 \mathrm{mmHg}$, Meyer et al. [9]. A good agreement between numerical and experimental results is obtained, confirming correct underlying physics of the two-way coupling between the blood flow and the "bad" cholesterol concentration under realistic biological conditions.

\section{Pulsating Blood Flow and Magnetic Particle Deposition in the Carotid Artery with Stenosis}

The following example addresses the pulsating blood flow within a patient-specific diseased carotid artery, as shown in Fig. 6a. This can be seen as a follow-up investigation of atherosclerosis development, but now, after the disease has become established. Here, we 


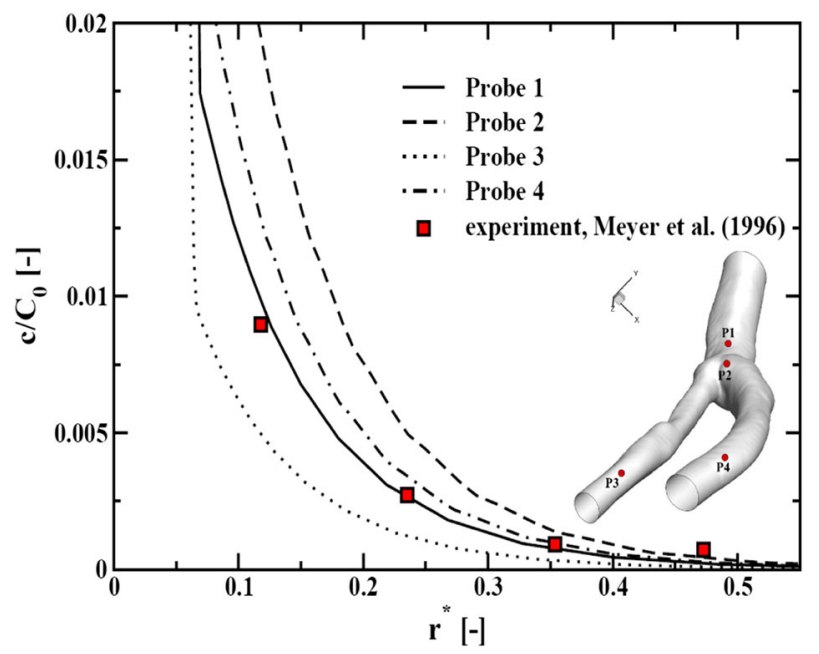

Fig. 5 The radial profiles of the non-dimensional LDL concentration $\left(c / C_{0}\right)$ within the interior of the artery wall (media) for some characteristic locations (P1-P2-P3-P4), which are shown in the zoom-in. Comparative assessment with experimental studies performed in the rabbit artery subjected to identical transmural pressure of $70 \mathrm{mmHg}$, Meyer et al. [9]

would like to explore the possibility of delivering a medical drug primarily to the stenotic regions (regions where narrowing or constrictions of the arterial inner surface, caused by atherosclerosis, are present). In addition to the patient-specific geometry, the characteristic pulsating flow rates at the inlet of the central-carotid-artery $\left(Q_{C C A}=1.1 \times 10^{-5} \mathrm{~m}^{3} / \mathrm{s}\right.$,
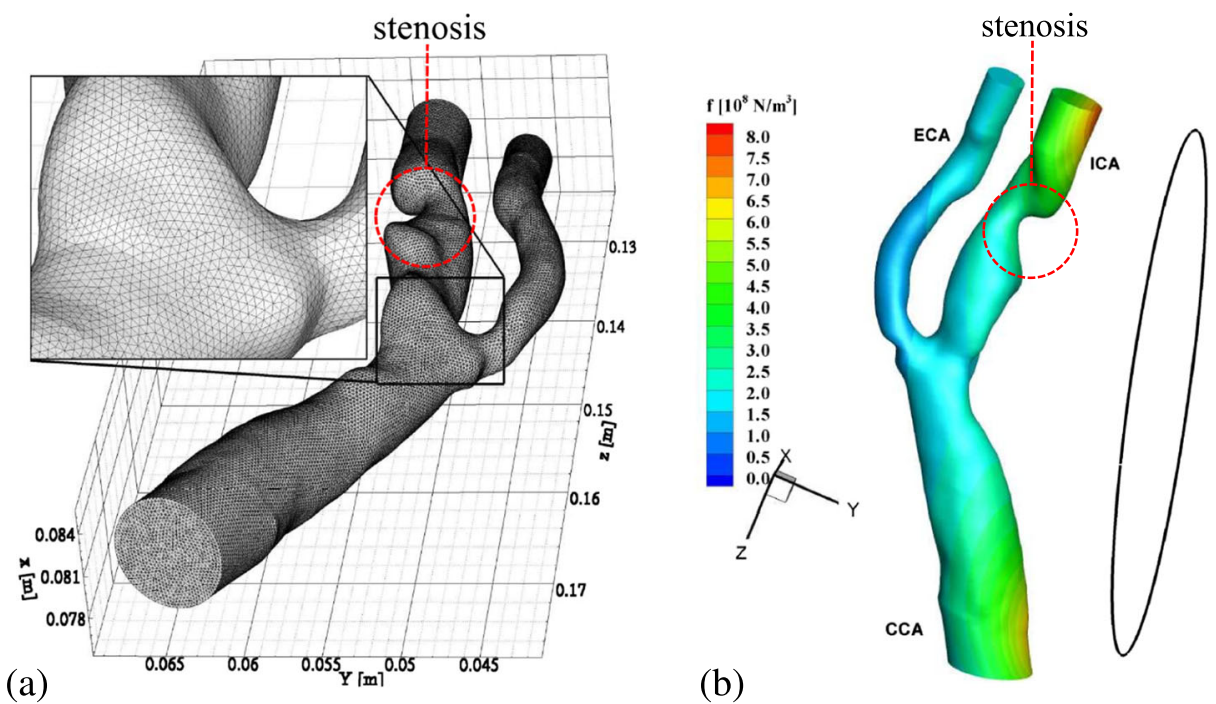

Fig. 6 a The numerical mesh used for the pulsating blood flow simulation in the patient-specific carotid artery bifurcation; $\mathbf{b}$ the spatial distribution of the imposed magnetization force field to steer magnetic drug carriers to the ICA bifurcation where the stenosis is located 
Holdsworth et al. [10]) and at the outlets (internal/external) of the carotid artery are specified as in previous case, i.e. $Q_{I C A}: Q_{E C A}=0.6: 0.4$, Groen et al. [8]. Due to the pulsating nature of the incoming velocity, flow exhibits a range of Reynolds numbers $(0 \leq \operatorname{Re} \leq 550)$, and it is important to include non-Newtonian behavior of the blood viscosity. Here, we adopted a generalized power law model of Ballyk et al. [11], which takes into account the shear-thinning of the whole blood caused by the red blood cell aggregation and dispersion at low shear rates that increases blood viscosity:

$$
\begin{aligned}
\mu(\dot{\gamma}) & =\lambda|\dot{\gamma}|^{n-1} \\
\lambda(\dot{\gamma}) & =\mu_{\infty}+\Delta \mu \exp \left[-\left(1+\frac{|\dot{\gamma}|}{a}\right) e^{-\frac{b}{|\dot{\gamma}|}}\right] \\
n(\dot{\gamma}) & =n_{\infty}-\Delta n \exp \left[-\left(1+\frac{|\dot{\gamma}|}{c}\right) e^{-\frac{d}{\mid \dot{\gamma}}}\right] \\
\dot{\gamma} & =\sqrt{S_{i j} S_{j i}}, \quad S_{i j}=\frac{1}{2}\left(\frac{\partial v_{i}}{\partial x_{j}}+\frac{\partial v_{j}}{\partial x_{i}}\right)
\end{aligned}
$$

where $\mu_{\infty}=0.0035 \mathrm{~Pa} \cdot \mathrm{s}, \Delta \mu=0.025 \mathrm{~Pa} \cdot \mathrm{s}, n_{\infty}=1, \Delta n=0.45, a=50 \mathrm{~s}^{-1}, b=3$ $\mathrm{s}^{-1}, c=50 \mathrm{~s}^{-1}, d=4 \mathrm{~s}^{-1}$, Ballyk et al. [11]. The numerical mesh is generated from the patient-specific surface geometry and contains approximately $1.2 \times 10^{6}$ tetrahedra, Fig. 6a. The second-order upwind discretization scheme is used for the convective terms of the momentum equation. The second-order fully implicit time-integration scheme with a time step of $\Delta t=0.005 \mathrm{~s}$ is used for the transient terms, [7]. The characteristic nondimensional parameters are Reynolds number $\left(16 \leq \operatorname{Re}=V_{0} D / v \leq 512\right)$ and Womersley number $\left(\alpha=D / 2(2 \pi f / \nu)^{1 / 2}=5.23\right)$.
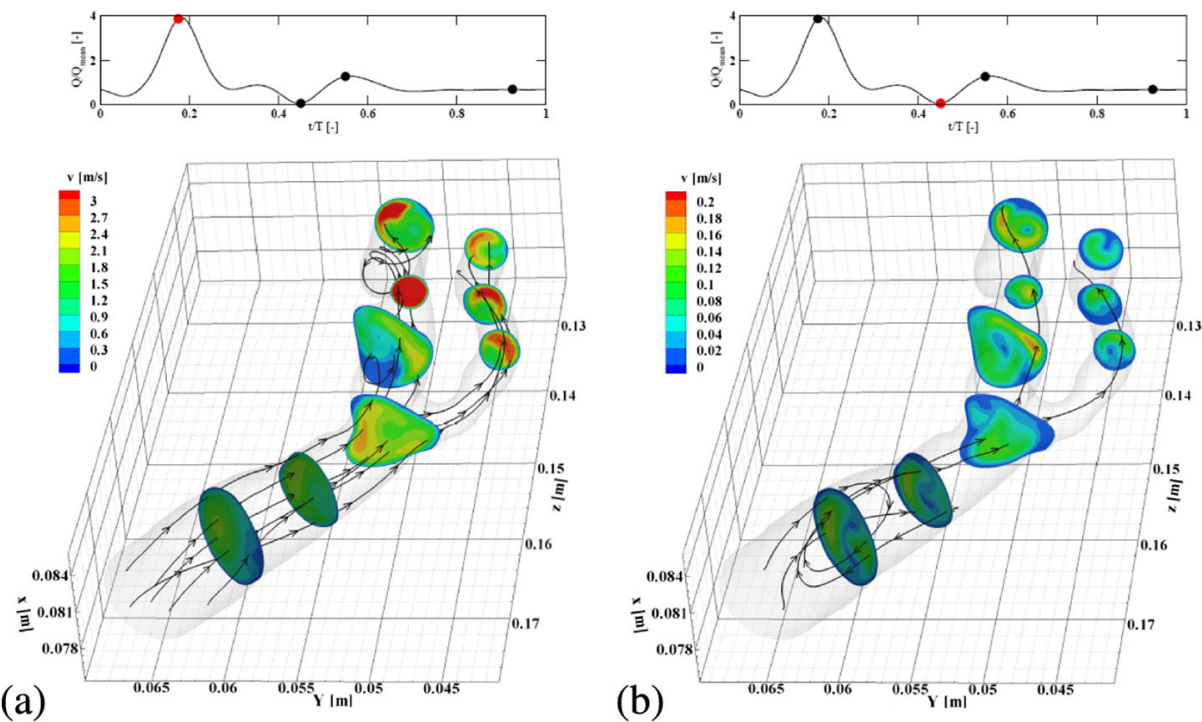

Fig. 7 The calculated flow patterns at two characteristic instantaneous positions during the cardiac cycle: (a) systole (the heart contraction, local maxima peak, $\mathrm{t}=0.175 \mathrm{~s}$ ) and (b) diastole (the heart relaxation, local minima peak, $\mathrm{t}=0.425 \mathrm{~s}$ ). The carotid artery flow rate as a function of time is specified as given in Holdsworth et al. [10] 


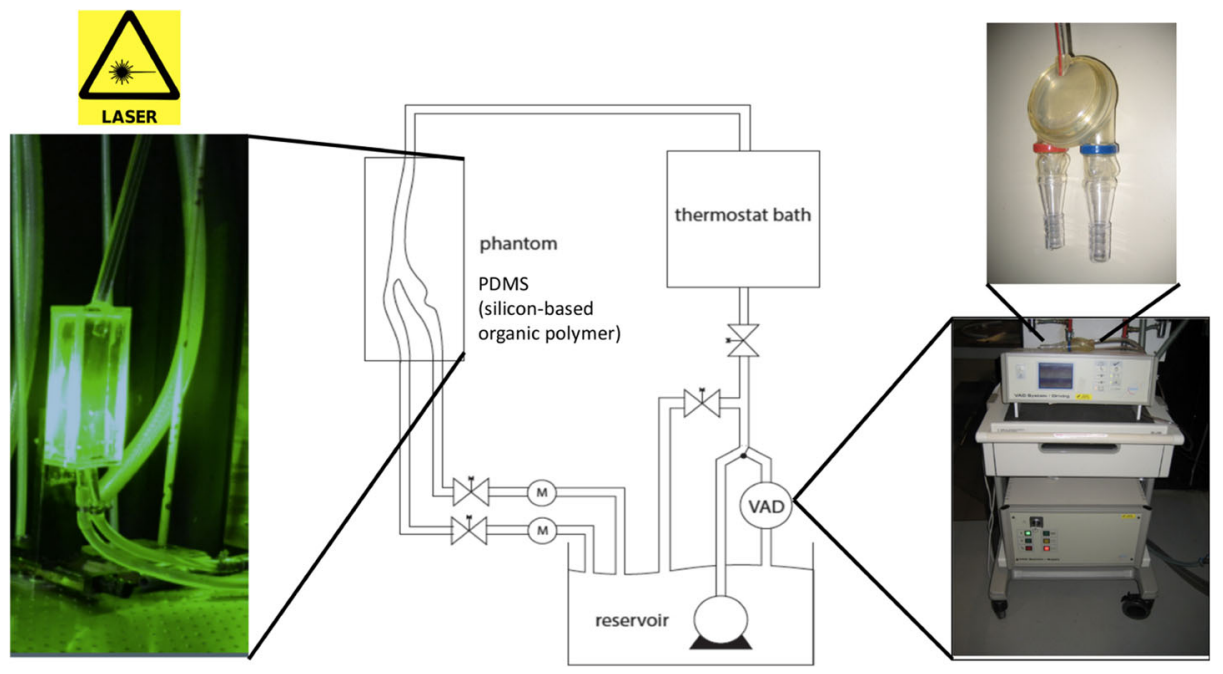

Fig. 8 The schematic of the experimental setup for PIV measurements of pulsating flow in the patientspecific carotid artery phantom. The laser illuminated PDMS phantom is shown on the left, whereas the ventricular assist device (VAD) is shown on the right

To get an impression of the significant changes in the blood flow patterns during the cardiac cycle, stream-traces and contours of the velocity magnitude at characteristic cross-sections are shown in Fig. 7. It can be seen that the stenosis in the internal-carotidartery generates the persistent recirculation zone (present during the entire pulsating cycle), Fig. 7a. In contrast to that, during the diastolic (the lowest flow rate), a significant flow reversal occurs in the proximity of the inlet plane of the central-carotid-artery, Fig. 7b. To check the accuracy of numerical simulations we performed also the PIV and MRI experimental studies. These measurements are carried out in the PDMS-built carotid-artery phantoms based on the rapid-prototyping technique, which makes it possible to construct models from the MRI or CT scans of the vascular systems of real patients. The schematic of the PIV experimental setup is shown in Fig. 8. A summary of the working fluid properties (water/glycerol mixture) and characteristic flow parameters are given in Table 1. The MRI

Table 1 Fluid properties and settings for PIV measurements

\begin{tabular}{lll}
\hline Parameter & Value & Unit \\
\hline Glycerol concentration & 64 & $\%$ by wt. \\
Dynamic viscosity & $1.5 \times 10^{-2}$ & Pa s \\
Density & 1165 & $\mathrm{~kg} / \mathrm{m}^{3}$ \\
Temperature of fluid & 25 & ${ }^{0} \mathrm{C}$ \\
Refractive index & 1.413 & - \\
Flow rate & $2-63$ & $\mathrm{ml} / \mathrm{s}$ \\
Outflow ratio ICA/ECA & $50 / 50,60 / 40$ & $\%$ \\
Inlet Reynolds number & $16-512$ & - \\
Womersley number & 5.23 & - \\
PIV particle size & $8-12$ & $\mu \mathrm{m}$ \\
\hline
\end{tabular}




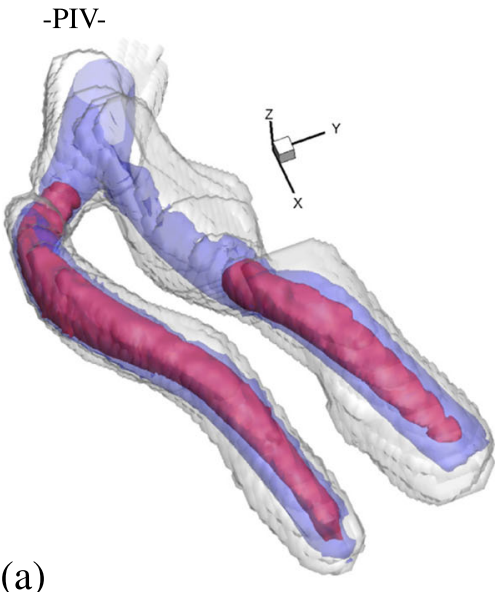

(a)

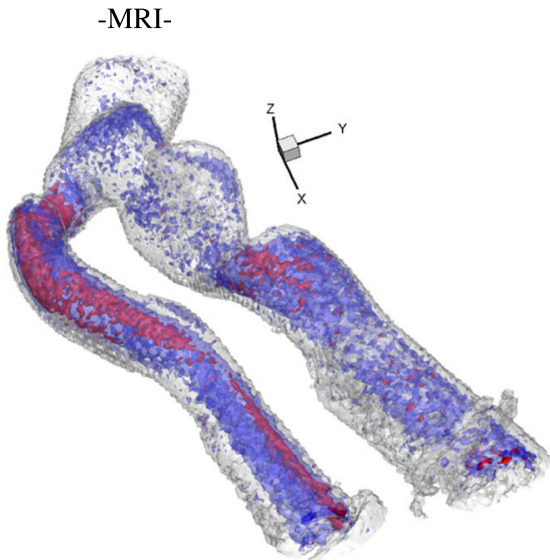

(b)

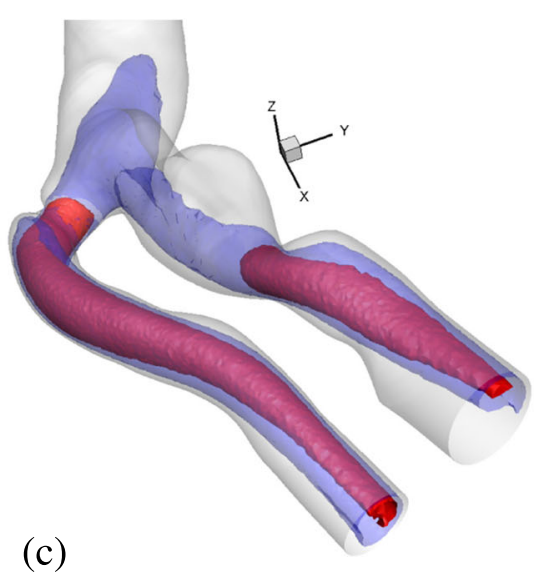

Fig. 9 Isosurfaces of the non-dimensional velocity magnitude $\left(|v| / v_{0}\right)$ for the steady flow conditions at $R e=284$ and outlet flow ratios of ICA:ECA $=0.5: 0.5 ;|v| / v_{0}=0$ (-grey), 2 (-blue) and 3.5 (-red). Note that for PIV (a), only two velocity components are used $\left(v_{x}\right.$ and $v_{y}$ ) (from a total of 21 cross-sections in the z-direction)

measurements were performed in Leiden University Medical Centre, Leiden, The Netherlands with the Philips Achieva $7 \mathrm{~T}$ whole body MRI scanner. Isosurfaces of the measured and simulated velocity magnitude are shown in Fig. 9. Note that for the PIV measurements, only two available velocity components $\left(v_{x}\right.$ and $v_{y}$ ) are used, measured in 21 characteristic planes (with $1 \mathrm{~mm}$ increments) along the z-coordinate direction. It can be seen that overall, very good agreement between the PIV (Fig. 9a) and CFD (Fig. 9c) is obtained, especially in regions where the flow acceleration takes place (indicated by red iso-surfaces), whereas MRI (Fig. 9b) was able to provide qualitatively reasonable results. Note that only standard clinical set-up working conditions of the MRI scanner have been used without addition of phase-contrast agents. The typical MRI scanning time was about $45 \mathrm{~min}$. Typical resolutions are 0.47:0.47 $\mathrm{mm}^{2}, 0.35: 0.35: 0.7 \mathrm{~mm}^{3}$ and 0.075:0.075:0.075 $\mathrm{mm}^{3}$ for PIV, MRI and CFD, respectively. 
-PIV-

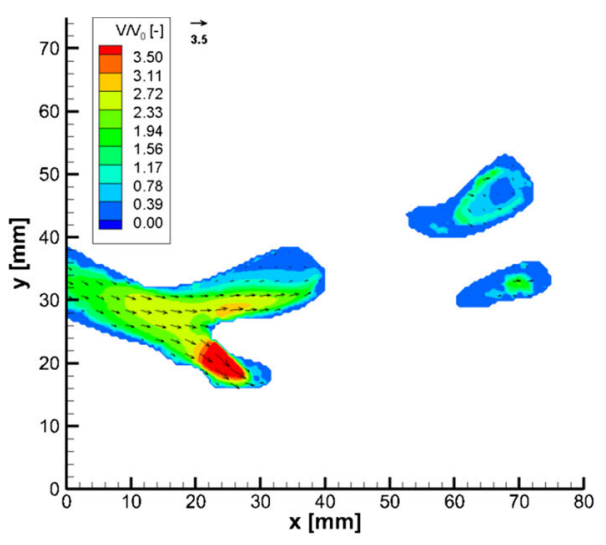

-MRI-

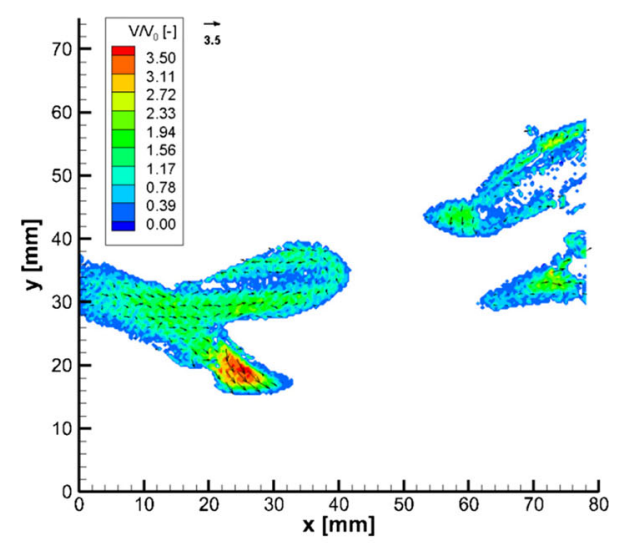

-CFD-

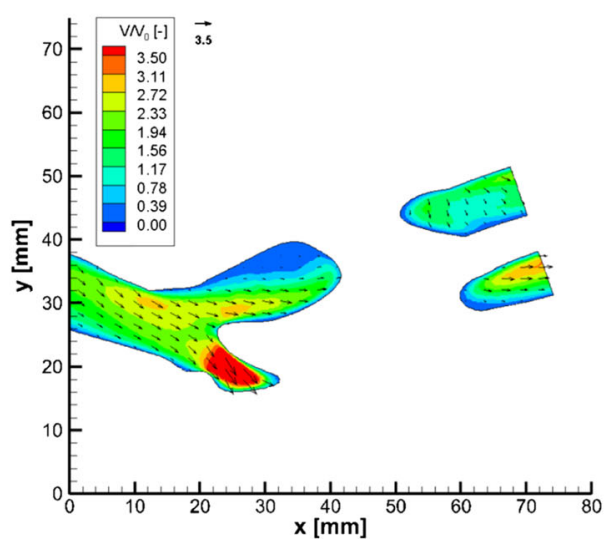

Fig. 10 Comparison between the PIV, MRI and CFD results in the characteristic horizontal cross-section for the patient-specific carotid artery shown in Fig. 6

More detailed comparisons are shown in Figs. 10 and 11, where contours and profiles of the non-dimensional velocity magnitude are shown. Exact locations at which profiles and contours are extracted are indicated in Fig. 11-top, where the blue mask indicates plane and A1-A2 lines indicate profiles. It can be seen that PIV and CFD show overall very good agreement and that MRI is able to provide qualitatively overall trends in the velocity profiles (especially at the A2 location, just after carotid bifurcation), Fig. $11 \mathrm{~b}$.

After obtaining reliable flow data, we investigate possibilities to impose a local delivery of the medical drug with magnetic cores. This is the essence of the Magnetic Drug Targeting (MDT) technique. The MDT showed very promising results in experimental studies on animals and in pre-clinical studies on human patients, e.g. Lübbe et al. [13, 14], Arnold and Parak [15], Torchilin [16]. In our previous numerical studies, we investigated various combinations of the MDT concept in simplified (Kenjereš [17], Haverkort et al. [18], Cohen Stuart et al. [19], Rusli et al. [20]) and complex (Haverkort et al. [21], Kenjereš and Righolt [22]) vascular geometries. For all cases, a significant improvement in local capturing of the magnetic particles is demonstrated, proving the potential of MDT. The MDT approach requires introduction of the spatial distribution of the imposed magnetic field (as shown in 

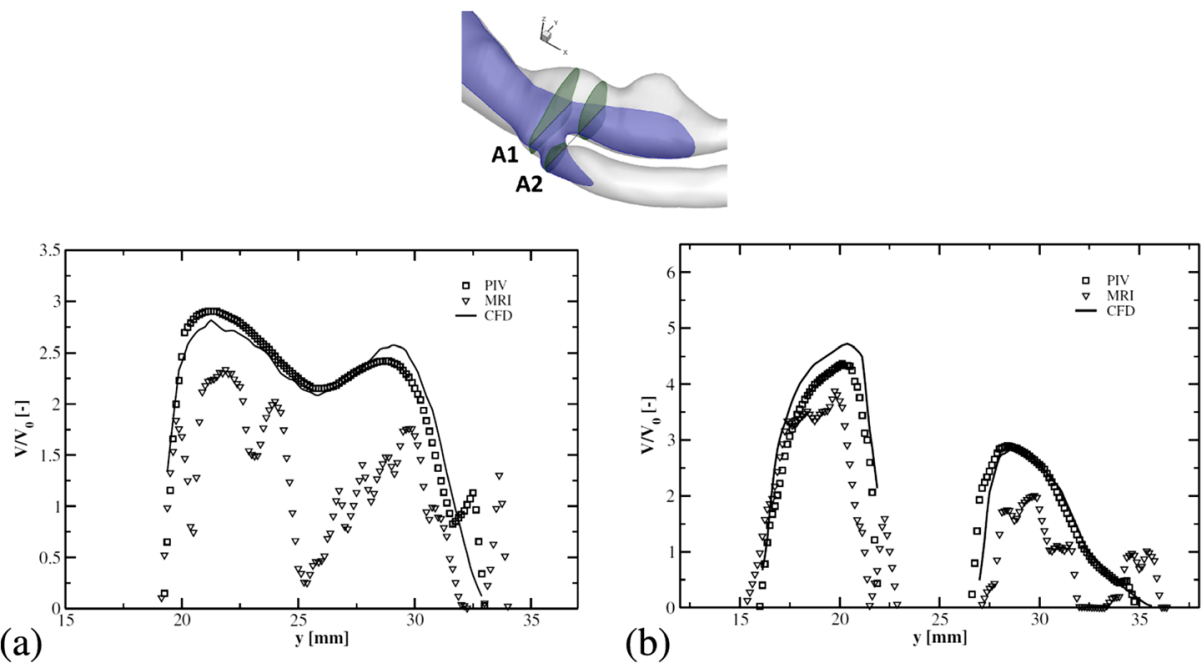

Fig. 11 Comparison of the measured (PIV and MRI) and numerical (CFD) velocity profiles at characteristic locations (a) before (A1) and (b) after (A2) carotid bifurcation for identical flow conditions as shown in Figs. 7 and 8

Fig. 6b) and additional equations for Lagrangian particle tracking. The latter is achieved by considering a balance of all active forces acting on particles:

$$
m_{p} \frac{\partial \mathbf{u}_{p}}{\partial t}=\mathbf{f}_{d}+\mathbf{f}_{v}+\mathbf{f}_{m}
$$

where $\mathbf{u}_{p}$ is the particle velocity, and $\mathbf{f}_{d}, \mathbf{f}_{v}$ and $\mathbf{f}_{m}$ are drag, virtual mass and magnetization force, respectively. The drag force is calculated as:

$$
\begin{aligned}
\mathbf{f}_{d} & =\frac{1}{2} \rho A_{\perp} C_{D}\left(\operatorname{Re}_{p}\right)\left|\mathbf{v}-\mathbf{u}_{p}\right|\left(\mathbf{v}-\mathbf{u}_{p}\right) \\
\operatorname{Re}_{p} & =\frac{\rho_{p} D_{p}\left|\mathbf{v}-\mathbf{u}_{p}\right|}{\mu}
\end{aligned}
$$

where $\rho_{p}, D_{p}$ and $A_{\perp}$ are the particle density, diameter and cross-section area, respectively. $C_{D}$ is a drag coefficient dependent on $\operatorname{Re}_{p}$, i.e. $C_{D}=24 / \operatorname{Re}_{p}$ for $\operatorname{Re}_{p}<0.1$, and $C_{D}=$ $3.69+22.73 / \operatorname{Re}_{p}+0.0903 / \operatorname{Re}_{p}^{2}$, for $0.1 \leq \operatorname{Re}_{p} \leq 1$, Morsi and Alexander [12]. The virtual, or added, mass force is calculated as:

$$
\mathbf{f}_{v}=\frac{1}{2} m_{p} \frac{\rho}{\rho_{p}} \frac{d}{d t}\left(\mathbf{v}-\mathbf{u}_{p}\right)
$$

and finally, the magnetic force is:

$$
\mathbf{f}_{m}=V_{m} \mu_{0} \mathbf{M} \cdot \nabla \mathbf{H},
$$

with $V_{m}$ the magnetic core volume, $\mu_{0}$ the permeability of free space, $\mathbf{M}$ the magnetization force per unit area and $\mathbf{H}$ the auxiliary magnetic field. For fully saturated super-paramagnetic particles, $\mathbf{M}=M_{\text {sat }} \mathbf{H} /|H|$, where $M_{\text {sat }}$ is the saturation magnetization. The auxiliary magnetic field can be obtained from Biot-Savart's law for different combinations of the electric current carriers (single/multiple straight wire, single/multiple loop-wire, etc.). Since a very diluted concentration of the injected medical drug is imposed, one-way coupling between particles dynamics and blood flow is assumed. 
The particles are uniformly injected at the inlet section of the CCA. The iron-core particles with saturation magnetization of $M_{\text {sat }}=10^{6} \mathrm{~A} / \mathrm{m}$ and with density of $\rho_{p}=6450$ $\mathrm{kg} / \mathrm{m}^{3}$ are considered. A range of particle diameters is investigated, i.e. $0.25 \leq d_{p} \leq 4 \mu \mathrm{m}$, where the largest value is determined by the characteristic diameter of the micro-capillary vascular system. This gives the following range of the non-dimensional Stokes numbers: $2 \times 10^{-7} \leq \mathrm{St}=\rho_{p} d_{p}^{2} v_{0} / 18 \mu D \leq 5.5 \times 10^{-5}$. The magnetic-particle number is introduced, which represents a ratio between the magnetization and drag forces $\left.\left(\mathrm{Mn}_{p}=f_{m} / f_{d}\right)\right)$. The 5th order Runge-Kutta time-integration scheme is used to solve Eq. 12. In addition to the total integral particle deposition, we also introduce a local particle deposition efficiency, which is calculated as:

$$
\zeta(\mathbf{x})=\frac{n_{\text {wall }}\left(\left|\mathbf{x}_{p, i}-\mathbf{x}\right|<\varepsilon\right)}{n_{\text {in }}}
$$

and represents the amount of particles deposited along the wall within a pre-specified neighborhood where $\varepsilon$ is the characteristic sample distance (usually $1 \mathrm{~mm}$ ). Here, $n_{\text {in }}$ is the total number of injected particles, $n_{\text {wall }}$ is the number of particles deposited along the wall, and $\mathbf{x}_{p, i}$ is the deposition location of the i-th particle. Two main conclusions can be drawn from Fig. 12. First, that it is possible to redirect the magnetic-core particles towards the internal carotid artery (where the stenotic region is present) and second, that local deposition efficiency of the particles with magnetic core can be significantly enhanced in comparison with particles without magnetic core. An overview of the local and cumulative deposition efficiencies for different classes of the particles is shown in Fig. 13. For the $d_{p}=4 \mu \mathrm{m}$ class of particles, the cumulative deposition efficiency shows an increase from 0.1 (no magneticcore) to $0.7\left(d_{p} / d_{m}=1.26\right)$, Fig. 13-bottom/left. For smaller particles $\left(d_{p}=1 \mu \mathrm{m}\right)$, the

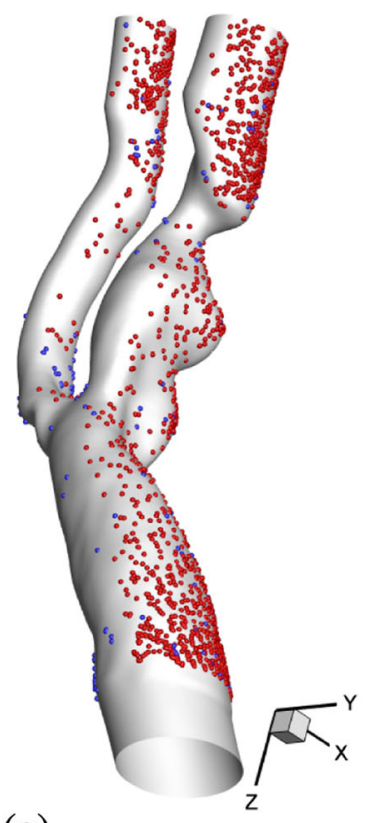

(a)

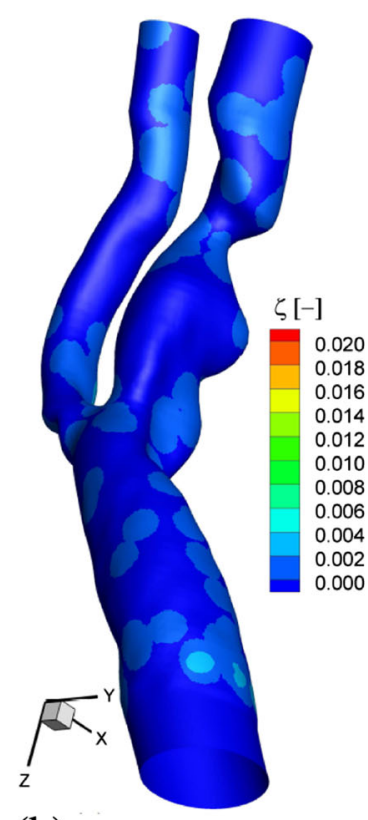

(b)

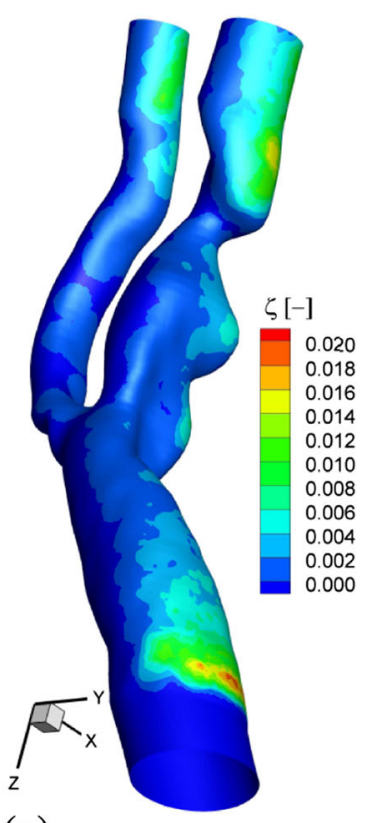

(c)

Fig. 12 a The scatter plot of particles $\left(d_{p}=d_{m}=2 \mu \mathrm{m}\right)$ deposited along the artery wall with (red) and without (blue) an imposed magnetic field $\left(\left|b_{0}\right|_{\max }=2 \mathrm{~T}\right)$; The contour plots of the local deposition efficiency ( $\zeta)$ (b) without and (c) with magnetic field 

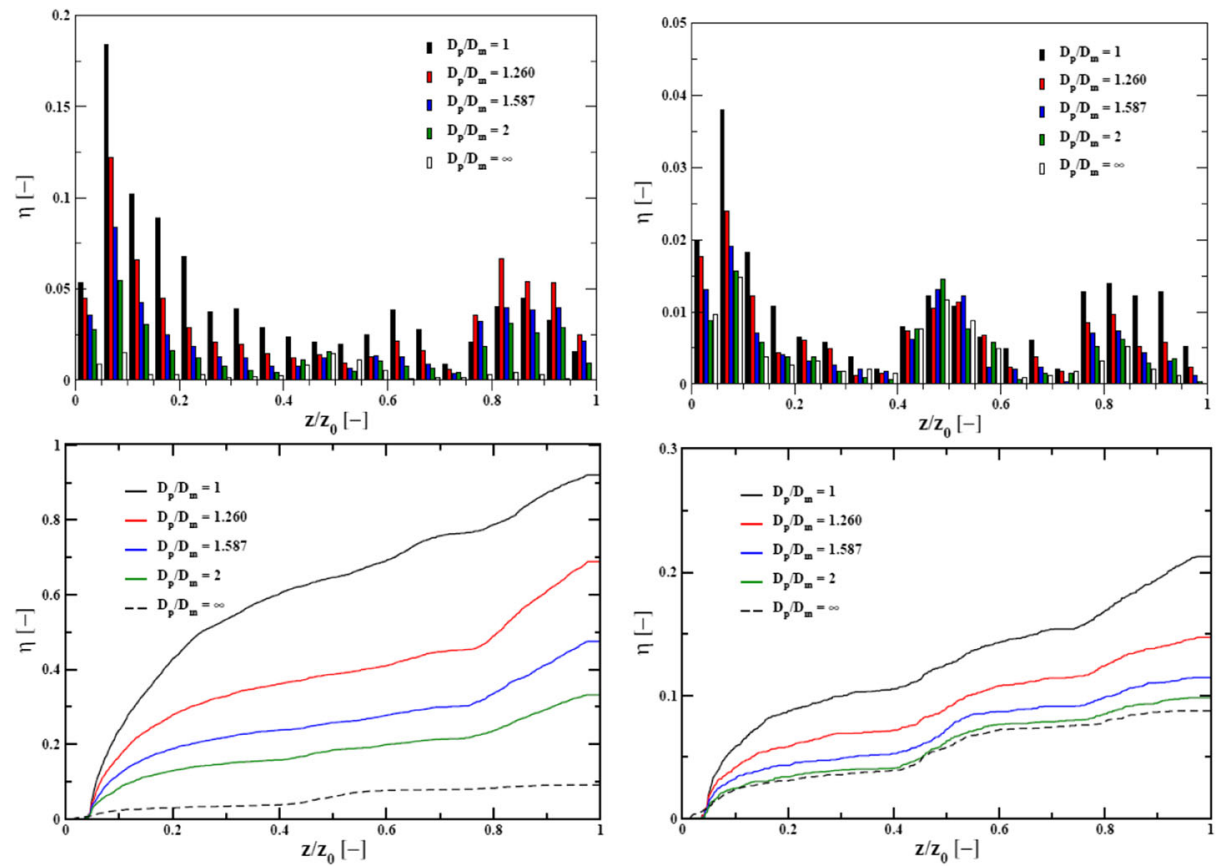

Fig. 13 The local (-top) and cumulative (-bottom) capture efficiency for the CA bifurcation and particles of $d_{p}=4 \mu \mathrm{m}$ (-left) and $1 \mu \mathrm{m}$ (-right) (external diameter) and different magnetic core diameters $\left(d_{m}\right)$ for an imposed magnetic field of $\left|b_{0}\right|_{\max }=2 \mathrm{~T}$ (shown in Fig. 5-right)

magnetic-capturing is less efficient but still significant, e.g. 0.09 (no magnetic core) versus 0.21 (with magnetic core), Fig. 13-bottom/right. From the standpoint of medical applications, it is particularly important to know the local deposition efficiency. Specifically, an estimation of the quantity of the injected medical drug delivered to the pre-defined region(s) is essential. For the carotid-artery bifurcation, the local deposition efficiency in the proximity of the stenosis $\left(z / z_{0} \approx 0.8\right)$ shows a more than eight-fold and three-fold increase for particles with magnetic core ratio of $d_{p} / d_{m}=1.26$, with $d_{p}=4 \mu \mathrm{m}$ and $d_{p}=1 \mu \mathrm{m}$, respectively.

It can be concluded that multi-phase simulations of the pulsating blood flow with injected magnetic-core particles subjected to an external magnetic field gradient, can be powerful tool for the patient-specific optimization of the local medical-drug delivery for advanced stages of cardiovascular diseases.

\section{Magnetic Control of Aerosol Distribution in the Human Respiratory System}

A dramatic increase of the respiratory diseases among children a result of exposure to increased environmental air pollution is reported in Chauhan and Johnston [23] and Ayres 


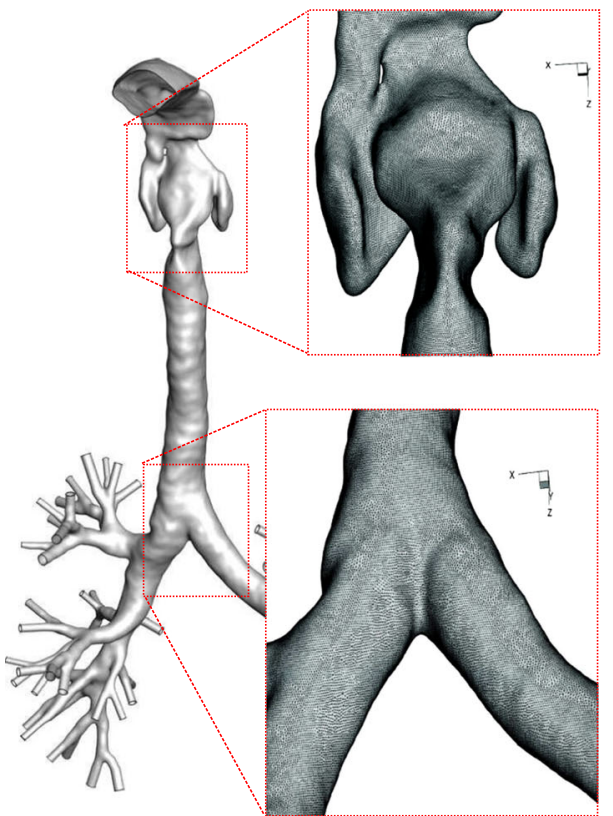

(a)

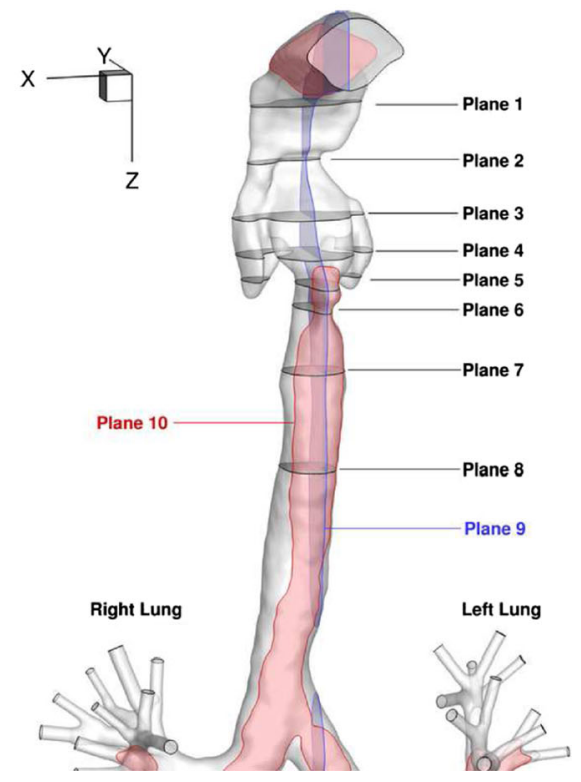

(b)

Fig. 14 a The geometry and zoom-ins of the numerical mesh (total of $1.5 \times 10^{7} \mathrm{CVs}$ ) of the upper-airways with up to 8 generation of the bronchioles bifurcations in the human respiratory system; $\mathbf{b}$ The characteristic planes where comparative assessment between experimental and numerical results is performed. Note that distance between Plane 1 and Plane 9 is approximately $0.155 \mathrm{~m}$

et al. [24]. Especially alarming is the evidence that exposure to outdoor air pollution causes lung cancer ${ }^{2}$. In that report, particulate matter (complex mixture of small particles and liquid droplets) was classified as carcinogenic to humans. Of particular concern are particles of $10 \mu \mathrm{m}$ or smaller, because of their ability to enter the lungs. Numerical simulations of the upper airways in humans can be a powerful tool for prediction of the distribution of different classes of inhaled particles. In addition, computer studies can improve different strategies to deliver medical drug aerosols to specific locations within the human respiratory system to fight respiratory diseases, Kleinstreuer et al. [25], Grotberg [26]. Dames et al. [27] reported on a novel experimental technique of targeted delivery of magnetic aerosol droplets to intact mice lungs. In these studies, it is demonstrated that by imposing sufficiently strong magnetic field gradients close to the main trachea bifurcation, steering of magnetic droplets towards

\footnotetext{
${ }^{2}$ World Health Organization, International Agency for Research on Cancer, Press release No.221, the 17th of October, 2013
} 


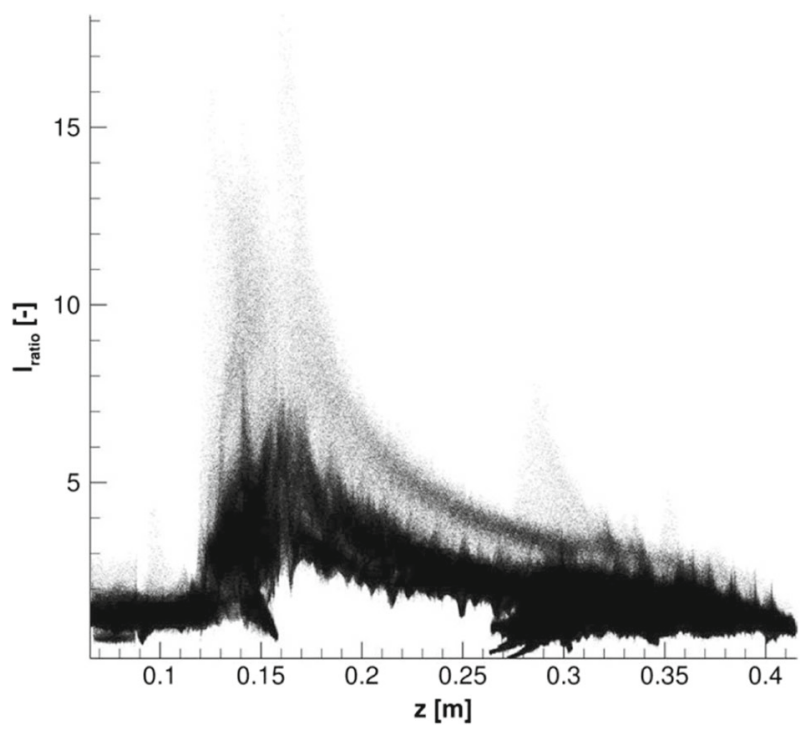

Fig. 15 The scatter-plot of the ratio between typical Kolmogorov length scales $\left(\eta_{k}=\left(v^{3} / \varepsilon\right)^{1 / 4}\right)$ and characteristic control-volume based length-scales $\left(\Delta V^{1 / 3}\right)$ for the numerical tetrahedral mesh shown in Fig. 13. The dissipation rate of turbulent kinetic energy $(\varepsilon)$ is obtained from the preliminary RANS-type simulation with SST model

the right lung can be achieved, resulting in an overall four-fold increase in the drug deposition compared to the neutral situation. In conclusion, Dames et al. [27] proposed further developments and scaling-up of the presented approach to human lungs. The next example in our study is drug-aerosol delivery in the human respiratory system, shown in Fig. 14a. In contrast to the complex blood-rheology that should account for the non-Newtonian effects for simulations of blood flow in cardiovascular system, here we are dealing with simple air as a working fluid. The modeling challenge here lies in the significantly higher values of Reynolds number (up to approximately 4500, for an inflow rate of $60 \mathrm{~L} / \mathrm{min}$ ), which in combination with the complex geometry of the human airway system, requires capturing the local turbulence generation. To achieve this, we applied two approaches: two-equation based eddy-viscosity RANS, and LES. The first one is employed because of its simplicity and relatively low computational costs. Moreover, the RANS results are used to make the preliminary estimates of the dissipation rate of the turbulent kinetic energy. This information is then used to properly redesign and redefine numerical mesh, which can be used for the reasonable resolved LES. The SST-Transient model is applied because of its suitability for flows where some transient effects can play a significant role, Menter et al. [28]. The steady inflow conditions of $60 \mathrm{~L} / \mathrm{min}$ that correspond to the top range of lung capacity during extensive exercise is considered. The scatter-plot of the characteristic ratio between typical Kolmogorov length-scales and the characteristic control-volume based length-scale $\left(\Delta V^{1 / 3}\right)$ is shown in Fig. 15.

This is done for a numerical mesh containing approximately $15 \times 10^{6}$ control volumes. It can be seen that except for the region around $\mathrm{z}=0.15 \mathrm{~m}$, this ratio is less than 5 , indicating 


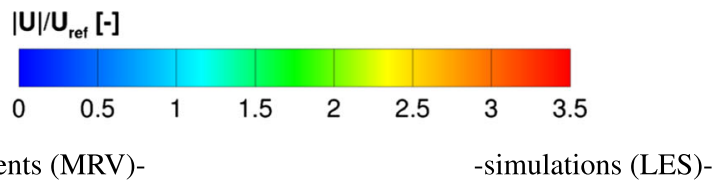

-experiments (MRV)-

-Plane 2-
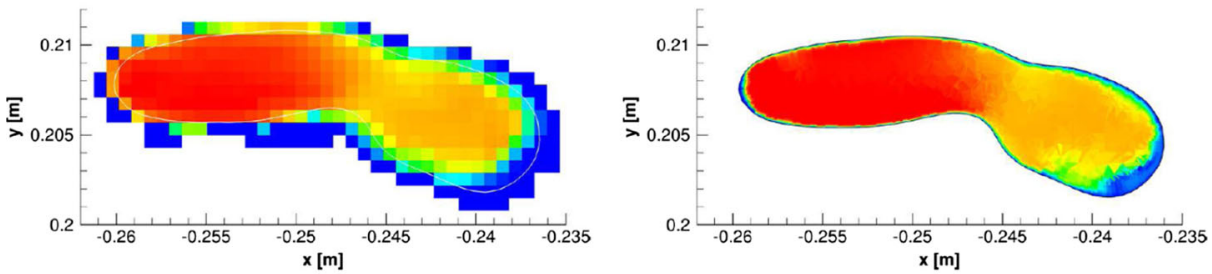

-Plane 4-
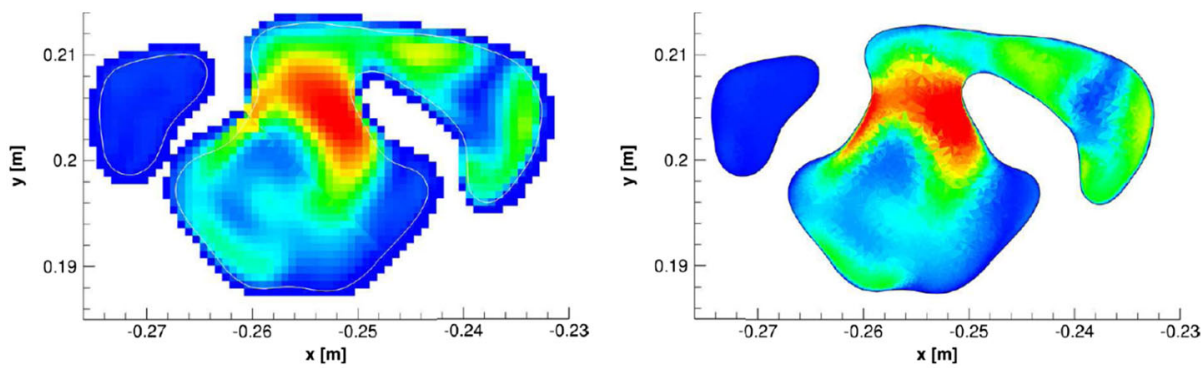

-Plane 8-
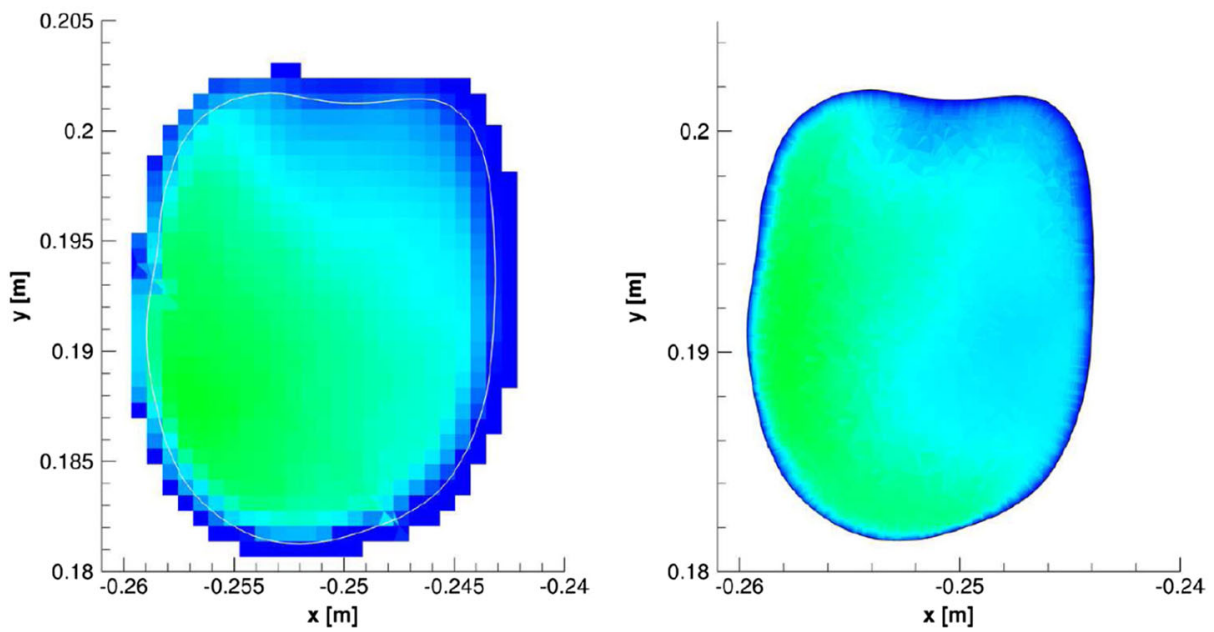

Fig. 16 Comparison between the water based MRV experiments (performed by the Stanford University group, Coletti et al. [30]) (-left) and LES-WALE simulations (-right) for contours of the velocity magnitude in characteristic planes shown in Fig. 14b: Plane2 ( $\mathrm{z}=0.1 \mathrm{~m})(-$ top $)$, Plane4 ( $\mathrm{z}=0.146 \mathrm{~m})(-$ middle) and Plane8 $(\mathrm{z}=0.21 \mathrm{~m})(-$ bottom $)$ 

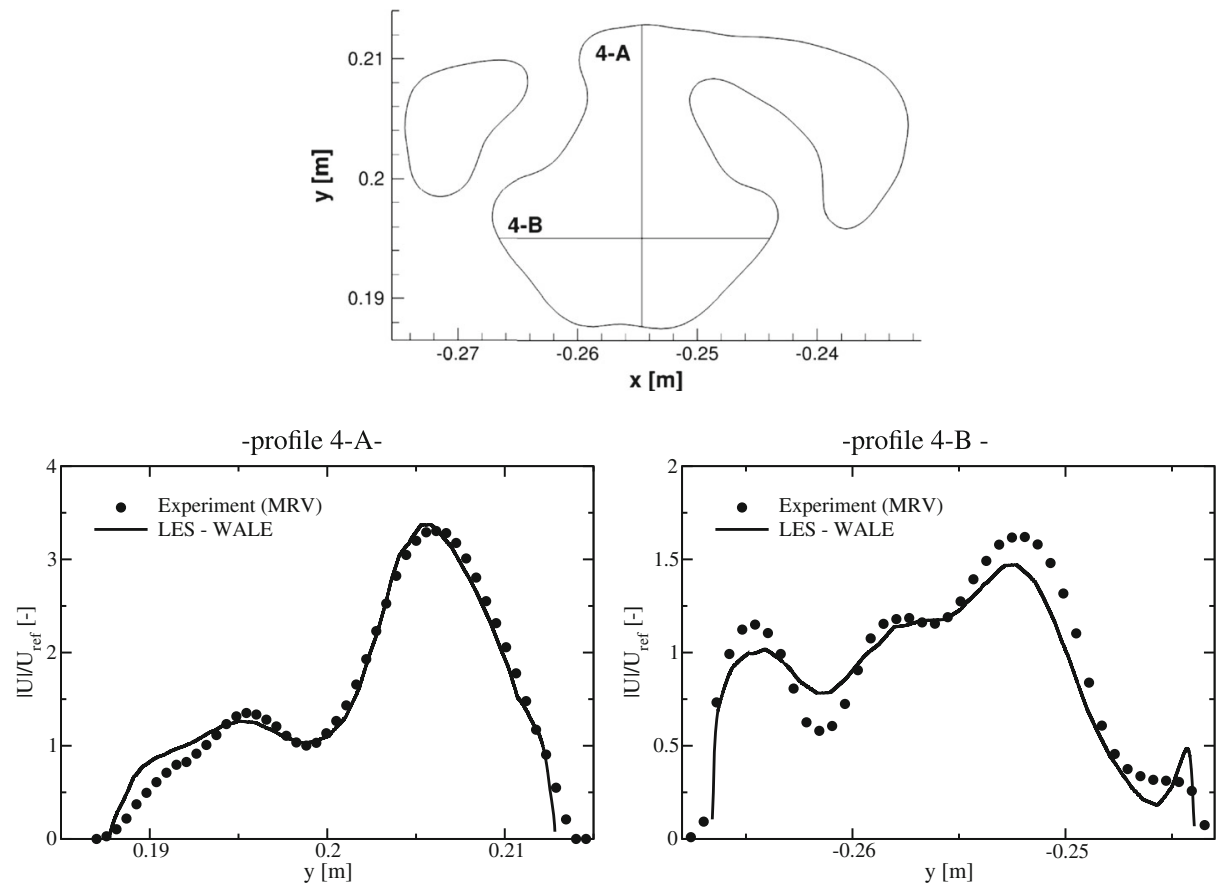

Fig. 17 Comparison of the characteristic profiles (along the 4A and 4B lines, in plane 4 shown in Fig. 14b, $\mathrm{z}=0.146 \mathrm{~m}$ ) of the velocity magnitude obtained in the MRV experiment (performed by the Stanford University group) and LES

a good mesh for LES. Here, we applied LES with the wall-adapting-local-eddy-viscosity (WALE) subgrid-closure, Nicoud and Ducros [29]:

$$
\begin{gathered}
v_{S G S}=L_{s}^{2} \frac{\left(s_{i j}^{d} s_{i j}^{d}\right)^{3 / 2}}{\left(\bar{s}_{i j} \bar{s}_{i j}\right)^{5 / 2}+\left(s_{i j}^{d} s_{i j}^{d}\right)^{5 / 4}}, \\
\bar{s}_{i j}=\frac{1}{2}\left(\frac{\partial v_{i}}{\partial x_{j}}+\frac{\partial v_{j}}{\partial x_{i}}\right), s_{i j}^{d}=\frac{1}{2}\left[\left(\frac{\partial v_{i}}{\partial x_{j}}\right)^{2}+\left(\frac{\partial v_{j}}{\partial x_{i}}\right)^{2}\right]-\frac{1}{3}\left(\frac{\partial v_{k}}{\partial x_{k}}\right)^{2} \delta_{i j}
\end{gathered}
$$

and where $L_{s}=\min \left(\kappa d, C_{w} \Delta\right)$, with $C_{w}=0.35, \kappa=0.41$ and $d$ as the distance to the closest wall. The time integration is performed with a fully implicit second-order scheme with characteristic time step of $\Delta t=10^{-4} \mathrm{~s}$, [7]. Comparison of the velocity magnitudes of the water-based magnetic resonance velocimetry (MRV) ${ }^{3}$ experiment and long-term averaged LES-WALE simulations in a few characteristic cross-sections (as indicated in Fig. 14b) is shown in Fig. 16b. It can be seen that very good agreement is obtained for both locations. In addition, more detailed comparisons of the velocity magnitude profiles are shown in Fig. 17. It can be concluded that LES simulation is in good agreement with MRV profiles for all

\footnotetext{
${ }^{3}$ The MRV experiments were performed by the Stanford University group using a 3T General Electric whole body scanner with a spatial resolution of $0.7 \mathrm{~mm}$, Coletti et al. [30].
} 

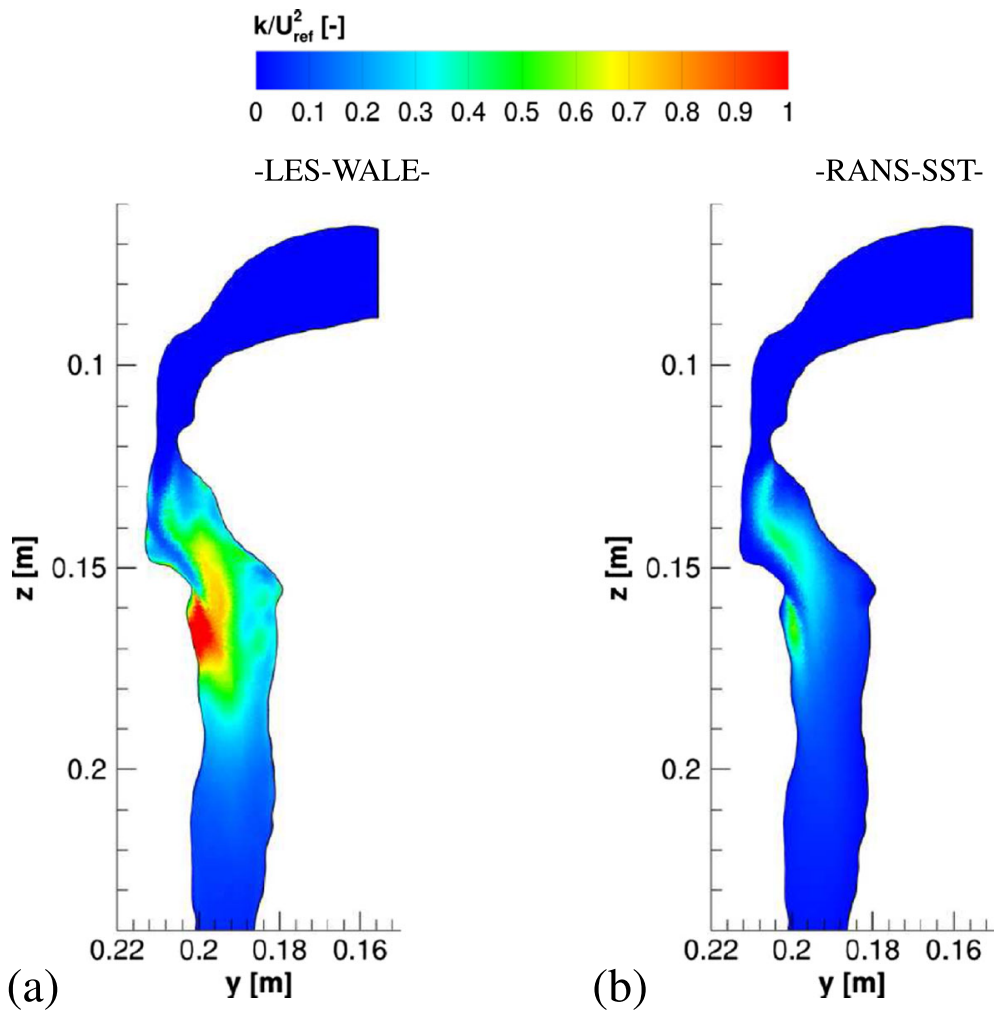

Fig. 18 Contours of the resolved turbulent kinetic energy in the central vertical plane (Plane 9, $\mathrm{x}=0.255 \mathrm{~m}$, shown in Fig. 13b ) obtained with LES-WALE [29] and RANS SST-Transient model, [28]

locations. The contours of the turbulence kinetic energy in the central vertical plane are shown in Fig. 18. It can be seen that turbulence is generated just after the oropharynx region
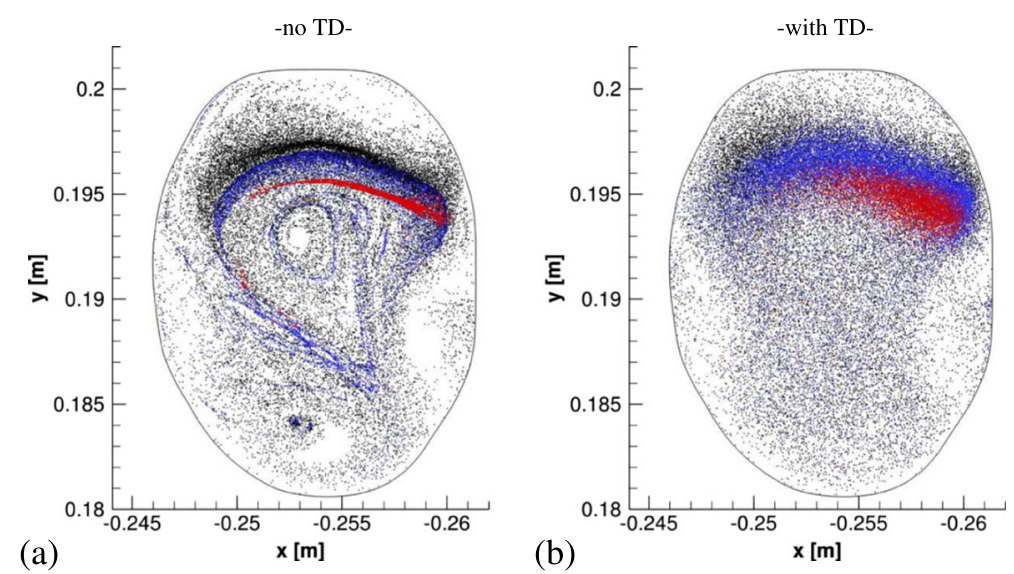

Fig. 19 Particle distributions at the plane $(\mathrm{z}=0.17 \mathrm{~m})$ shown in Fig. 13b, without (a) and with (b) turbulent dispersion term: $\mathrm{St}=4.8 \times 10^{-5}$ (black), $4.3 \times 10^{-2}$ (blue) and $1.2 \times 10^{-1}$ (red), which correspond to $d_{p}=0.1$, 3 and $5 \mu \mathrm{m}$, respectively 


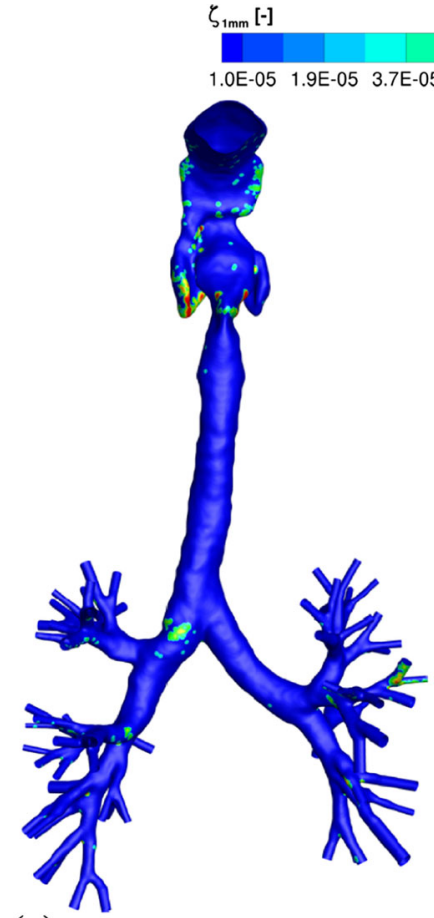

(a)

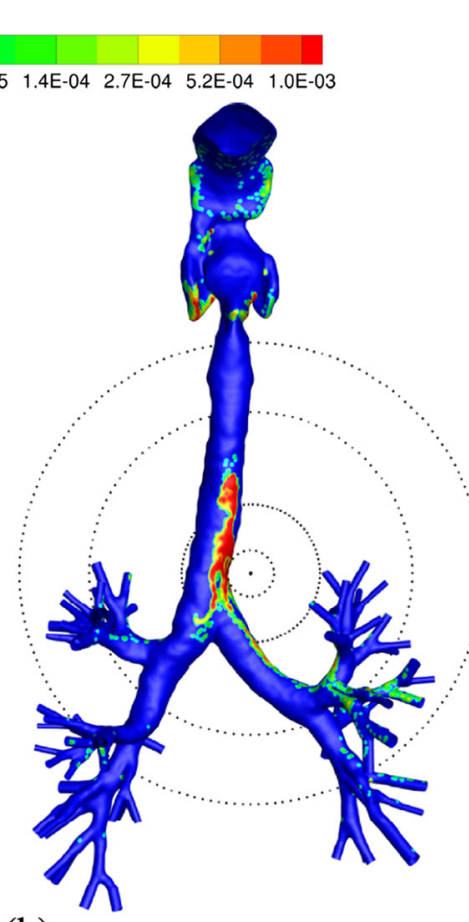

(b)

Fig. 20 Contours of the local deposition efficiency $(\zeta)$ in the upper-airways human respiratory system for the magnetic-core particles $\left(d_{\mathrm{p}}=3 \mu \mathrm{m}, \mathrm{St}=4.3 \times 10^{-2}, \mathrm{Mn}_{\mathrm{p}}=1.7 \times 10^{-1}\right)$ without $(\mathbf{a})$ and with imposed magnetic field gradient (b)

(the narrowing bend) and that it decays quite rapidly after that. It is also noted that LES levels of the turbulence kinetic energy are significantly higher than that of RANS which can have consequences when the drug-aerosol distribution within the human respiratory system needs to be addressed. This is illustrated in Fig. 19, where distributions of injected particles in the characteristic cross-section (Plane A), with and without turbulent dispersion terms, are compared. It can be seen that turbulent dispersion produces more homogeneous distributions.

Contours of the local deposition efficiency $\left(\zeta_{1 \mathrm{~mm}}\right.$, as defined in Eq. 17) for the $\mathrm{St}=4.3 \times$ $10^{-2}$ class of particles are shown in Fig. 20. Situations with particles without and with magnetic cores are considered for an imposed magnetic field gradient (the current-carrying wire), as well as whether the influence of the turbulent dispersion is included or not. It can be concluded that a significantly higher deposition efficiency is obtained for the magnetically affected particles. Also, the steering of the magnetic particles towards the left side of lungs can be effectively achieved. An overview of the total deposition efficiencies for different values of St for simplified and real human upper-airways geometries is shown in Fig. 21-top. It can be seen that the present simulations agree very well with similar experimental Cheng et al. ([31]) and numerical simulations Kleinstreuer et al. [25] in literature. The effects of the imposed magnetic field on capturing of the magnetic-particles are shown in Fig. 21-bottom. 

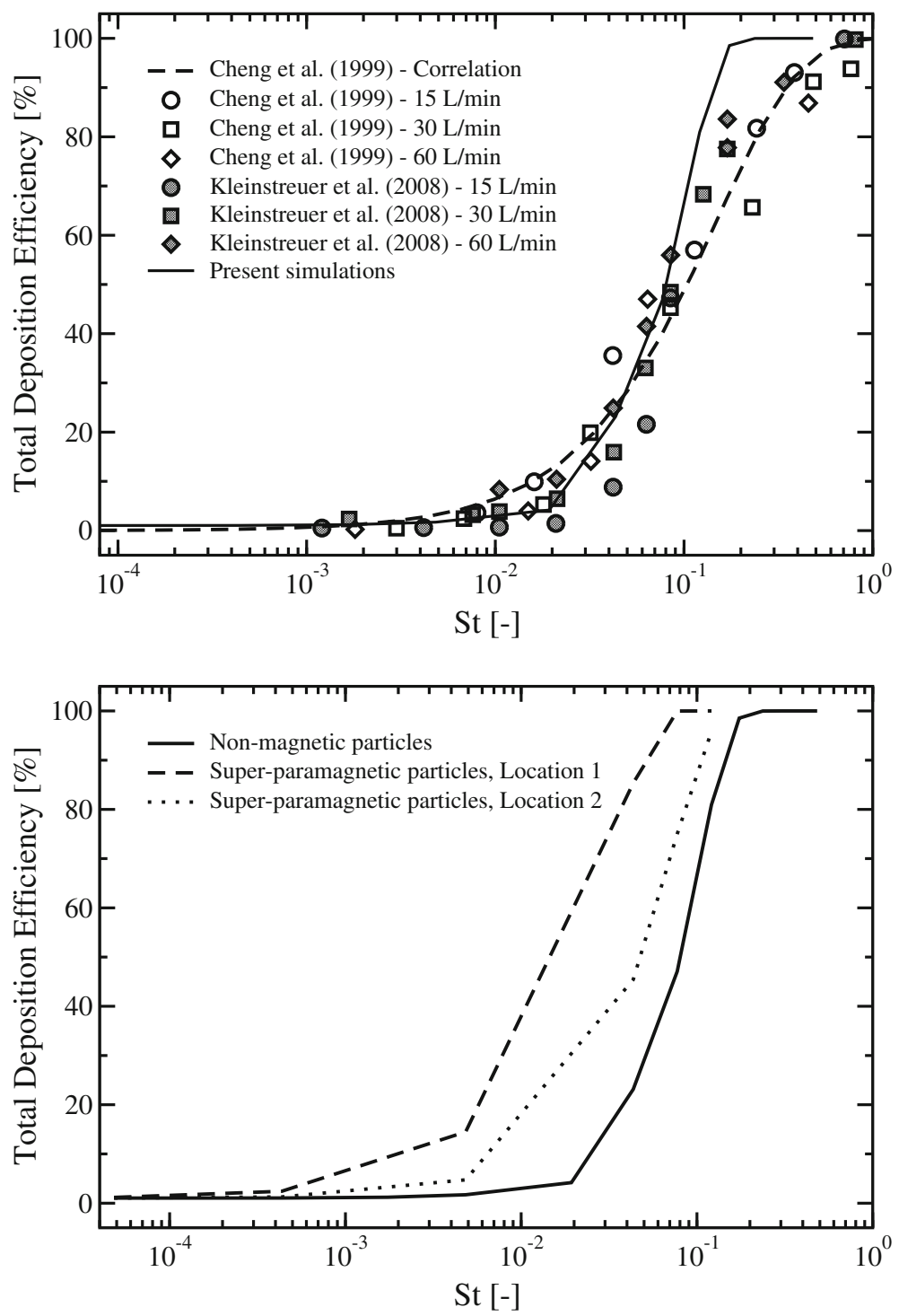

Fig. 21 The total deposition efficiency for different classes of particles (top- non-magnetic core; bottommagnetic core) within the upper airways of the human respiratory system shown in Fig. 13. Comparison with some similar studies in literature performed on simplified geometries of the upper airways, Cheng et al. [31] and Kleinstreuer et al. [25]. Two characteristic locations of the current-carrying wire are considered: Location $1, x=-0.252 \mathrm{~m}, \mathrm{z}=0.269 \mathrm{~m}$, distance $\mathrm{y}=0.01 \mathrm{~m}$; Location $2, \mathrm{x}=-0.252 \mathrm{~m}, \mathrm{z}=0.269 \mathrm{~m}$, distance $\mathrm{y}=0.1$ $\mathrm{m}$ (note that coordinate system is shown in Fig. 18 and that Location 1 is depicted in Fig. 20b)

The most significant enhancement is observed in $5 \times 10^{-4} \leq \mathrm{St} \leq 5 \times 10^{-2}$ range. For example, for $\mathrm{St}=0.01$, the five- and ten-fold increases are obtained for two configurations of the imposed magnetic field. 
In conclusion, it is demonstrated that LES proved to provide velocity predictions in excellent agreement with MRV measurements of the upper airways of the human respiratory system. Also, it is shown that the magnetic-drag-targeting concept can be successfully applied for this geometry as well.

\section{Conclusions and Outlook}

We demonstrated that it is possible to perform advanced numerical simulations of the blood or air flow in patient-specific configurations. The first example addressed blood-flow simulation in the healthy and diseased patient-specific carotid artery bifurcations. A recently developed mathematical model of the LDL and plasma distribution through the multi-layer structure of the arterial wall was successfully coupled with the blood-flow predictions within the carotid artery bifurcation. By comparing results with experimental results of LDL distributions within the arterial wall of a rabbit artery under identical transmural pressure conditions, it is concluded that the present version of the model can be used to predict locations within the vascular system where atherosclerosis can develop. In the second example, we studied pulsating blood flow within diseased (advanced atherosclerosis stage) carotid artery, and tested concept of magnetic drug targeting for local delivery of the medical drug in the proximity of the stenotic (narrowing) regions. To provide an extensive dataset for validation of the numerical simulations, we performed a series of experimental PIV and MRI measurements. A good agreement between numerical simulations and experiments is obtained in predicting both temporal and spatial variations of the velocity of blood during the cardiac cycle. It is shown that application of the MDT technique can lead to significant enhancement of the local deposition efficiency of the medical drug in the proximity of diseased regions (up to ten-fold increase in comparison with the neutral case).

Finally, distributions of drug aerosols inside the upper airways of the complex human respiratory system (from the mouth to the eight bronchial generation) are performed. Special attention is devoted to proper capturing of the locally generated turbulence, which has significant impact on aerosols distribution in comparison with a laminar regime. The results of the LES with the wall-adapting local eddy-viscosity model (WALE) were in excellent agreement with MRV experiments performed in the scaled water-model. It was demonstrated that the concept of the magnetic steering can also be applied for the real scale human-respiratory system.

Future applications of combined experimental (stereoscopic PIV, PC-MRI and ultrasound measurements) and numerical studies in dealing with complex advanced physical transport phenomena in simplified generic and complex patient-specific geometries can lead to improvements of localized medical drug delivery, which can significantly increase drug concentrations at the targeted regions. Only integrated applications of the in vivo (living systems), in vitro (laboratory condition) and in silico (computer simulation) studies can bring insight to key aspects of the underlying biology and physics of localized medical drug delivery in the human circulatory and respiratory systems.

Acknowledgments In the first place, I would like to acknowledge direct contributions from the 'MDT' team of $\mathrm{PhD}$ and MSc students from the former Department of Multi-Scale Physics and current Transport Phenomena Section, at the Delft University of Technology that made this exciting research possible, and which results are shown in this paper: Alexander de Loor (modeling and simulations of atherosclerosis), Bernhard Righolt (CFD of the MDT in carotid artery bifurcation), Gidi van de Belt and Jasper van der Starre (for experimental studies in CA bifurcations), Jimmy Leroy Tjin (for simulations of aerosol distributions in human respiratory system). Dr. Frank Gijsen, Biomechanic Laboratory of the Erasmus Medical Centre 
in Rotterdam, for providing geometries of the patient-specific carotid artery bifurcations and for inspiring discussions. Dr. Thijs van Osch, Radiology Department of Leiden University Medical Centre, for his support in performing MRI measurements of the carotid bifurcations. Dr. Fillipo Coletti, Department of Mechanical Engineering, Stanford University, USA, for kindly providing unpublished MRV measurements of the flow in human respiratory system. I gratefully acknowledge assistance in several forms from a consortium of lifesciences and medical-technology partners in South Holland (Delft, Rotterdam, Leiden), within Medical Delta (http://www.medicaldelta.nl) framework.

Open Access This article is distributed under the terms of the Creative Commons Attribution 4.0 International License (http://creativecommons.org/licenses/by/4.0/), which permits unrestricted use, distribution, and reproduction in any medium, provided you give appropriate credit to the original author(s) and the source, provide a link to the Creative Commons license, and indicate if changes were made.

\section{References}

1. Truskey, G.A., Yuan, F., Katz, D.F.: Transport phenomena in biological systems, 2nd Edition, pearson prentice hall bioengineering (2009)

2. Tarbell, J.M.: Mass transport in arteries and the localization of atherosclerosis. Annu. Rev. Biomed. Eng. 5, 79-118 (2003)

3. Yang, N., Vafai, K.: Modelling of low-density lipoprotein (LDL) transport in the artery-effects of hypertension. Int. J. Heat and Mass Transfer 49, 850-867 (2006)

4. Ai, L., Vafai, K.: A coupling model for macromolecule transport in a stenosed arterial wall. Int. J. Heat and Mass Transfer 49, 1568-1591 (2006)

5. Olgac, U., Poulikakos, D., Saur, S.C., Alkadhi, H., Kurtcuoglu, V.: Patient-specific three-dimensional simulation of LDL accumulation in a human left coronary artery in its healthy and atherosclerotic states. Am. J. Physiol. Heart Circ. Physiol. 296, H1969-H1982 (2009)

6. Kenjereš, S., de Loor, A.: Modelling and simulation of low-density lipoprotein transport through multilayered wall of an anatomically realistic carotid artery bifurcation. J. R. Soc. Interface. 11(91), 1-13 (2014). Art. No. 20130941

7. Academic Research, A.N.S.Y.S.: Release 15.0 ANSYS Inc (2014)

8. Groen, H.C., Simons, L., van den Bouwhuijsen, Q.J.A., Bosboom, E.M.H., Gijsen, F.H.J., van der Giessen, A.G., van de Vosse, F.N., Hofman, A., van der Steen, A.F.W., Witteman, J.C.M., van der Lugt, A., Wentzel J. J.: MRI-based quantification of outflow boundary conditions for computational fluid dynamics of stenosed human carotid arteries. J. Biomech. 43(12), 2332-2338 (2010)

9. Meyer, G., Merval, R., Tedgui, A.: Effects of pressure-induced stretch and convection on low-density lipoprotein and albumin uptake in the rabbit aortic wall. Circ. Res. 79(3), 532-540 (1996)

10. Holdsworth, D.W., Norley, C.J.D., Frayne, R., Steinman, D.A., Rutt, B.K.: Characterization of common carotid artery blood-flow waveforms in normal human subjects. Physiol. Meas 20, 219-240 (1999)

11. Ballyk, P.D., Steinman, D.A., Ethier, C.R.: Simulation of non-Newtonian blood-flow in an end-to-side anastomosis. Biorheology 31(5), 565-586 (1994)

12. Morsi, S.A., Alexander, A.J.: Investigation of particle trajectories in 2-phase flow systems. J. Fluid Mech. 55(2), 193-208 (1972)

13. Lübbe, A.S., Bergemann, C., Riess, H., Schriever, F., Reichardt, P., Possinger, K., Matthias, M., Dorken, B., Herrmann, F., Gurtler, R., Hohenberger, P., Haas, N., Sohr, R., Sander, B., Lemke, A.J., Ohlendorf, D., Huhnt, W., Huhn, D.: Clinical experiences with magnetic drag targeting: A phase I study with 4'-epidoxorubicin in 14 patients with advanced solid tumors. Cancer Res. 56(20), 4686-4693 (1996a)

14. Lübbe, A.S., Bergemann, C., Huhnt, W., Fricke, T., Riess, H., Brock, J.W., Huhn, D.: Cinical experiences with magnetic drug targeting: Tolerance and efficacy. Cancer Res. 56(20), 4694-4701 (1996b)

15. Alexiou, C., Schmid, R.J., Jurgons, R., Kremer, M., Wanner, G., Bergemann, C., Huenges, E., Nawroth, T., Arnold, W., Parak, F.G.: Targeting cancer cells: magnetic nanoparticles as drug carriers. Eur. Biophys. J. 35(5), 446-450 (2006)

16. Torchilin, V.P.: Nanoparticulates as drug carriers. Imperial College Press, London (2006)

17. Kenjereš, S.: Numerical analysis of blood flow in realistic arteries subjected to strong non-uniform magnetic fields. Int. J. Heat and Fluid Flow 29(3), 752-764 (2008) 
18. Haverkort, J.W., Kenjereš, S., Kleijn, C.R.: Magnetic particle motion in a Poiseuille flow. Phys. Rev. E 80(1), 1-12 (2009). Art. No. 016302

19. Cohen Stuart, D.C., Kleijn, C.R., Kenjereš, S.: An efficient and robust method for Lagrangian magnetic particle tracking in fluid flow simulations on unstructured grids. Computer and Fluids 40(1), 188-194 (2011)

20. Rusli, N., Kueh, A.B.H., Kenjereš, S.: Magnetic field effects on 3D blood flow patterns of straight and stenotic arteries. Advanced Science Latters 19(9), 2690-2693 (2013)

21. Haverkort, J.W., Kenjereš, S., Kleijn, C.R.: Computational simulations of magnetic particle capture in arterial flows. Ann. Biomed. Eng. 37(12), 2436-2448 (2009)

22. Kenjereš, S., Righolt, B.W.: Simulations of magnetic capturing of drug carriers in the brain vascular system. Int. J. Heat and Fluid Flow 35(3), 68-75 (2012)

23. Chauhan, A.J., Johnston, S.L.: Air pollution and infection in respiratory illness. Br. Med. Bull. 68, 95$112(2003)$

24. Ayres, J., Maynard, R., Richards, R.: Air Pollution and Health, Air Pollution Reviews -vol. 3. Imperial College Press, London (2006)

25. Kleinstreuer, C., Zhang, Z., Donohue, J.F.: Targeted drug-aerosol delivery in the human respiratory system. Annu. Rev. Biomed. Eng. 10, 195-220 (2008)

26. Grotberg, J.B.: Respiratory fluid mechanics. Phys. Fluids 23(021301), 1-15 (2011)

27. Dames, P., Gleich, B., Flemmer, A., Hajek, K., Seidl, N., Wiekhorst, F., Eberbeck, D., Bittmann, I., Bergemann, C., Weyh, T., Trahms, L., Rosenecker, J., Rudolph, C.: Targeted delivery of magnetic aerosol droplets to the lung. Nat. Nanotechnol. 2(8), 495-499 (2007)

28. Menter, F.R., Langtry, R., Völker, S.: Transition modelling for general purpose CFD codes. Flow Turbulence and Combustion 77(1-4), 277-303 (2006)

29. Nicoud, F., Ducros, F.: Subgrid-scale stress modelling based on the square of the velocity gradient tensor. Flow Turbulence and Combustion 62, 183-200 (1999)

30. Colleti, F., et al.: private communication (2014)

31. Cheng, Y.S., Zhou, Y., Chen, B.T.: Particle deposition in a cast of human oral airways. Aerosol Sci. Technol. 31, 286-300 (1999) 